TRANSACTIONS OF THE

AMERICAN MATHEMATICAL SOCIETY

Volume 363, Number 8, August 2011, Pages 4081-4107

S 0002-9947(2011)05187-1

Article electronically published on March 21, 2011

\title{
SPLITTING AND GLUING LEMMAS FOR GEODESICALLY EQUIVALENT PSEUDO-RIEMANNIAN METRICS
}

\author{
ALEXEY V. BOLSINOV AND VLADIMIR S. MATVEEV
}

\begin{abstract}
Two metrics $g$ and $\bar{g}$ are geodesically equivalent if they share the same (unparameterized) geodesics. We introduce two constructions that allow one to reduce many natural problems related to geodesically equivalent metrics, such as the classification of local normal forms and the Lie problem (the description of projective vector fields), to the case when the $(1,1)$-tensor $G_{j}^{i}:=g^{i k} \bar{g}_{k j}$ has one real eigenvalue, or two complex conjugate eigenvalues, and give first applications. As a part of the proof of the main result, we generalise the Topalov-Sinjukov (hierarchy) Theorem for pseudo-Riemannian metrics.
\end{abstract}

\section{INTRODUCTION}

Definition 1. Let $g$ and $\bar{g}$ be Riemannian or pseudo-Riemannian metrics on the same manifold $M^{n}$. We say that they are geodesically equivalent (notation: $g \sim \bar{g}$ ) if they have the same geodesics considered as unparameterized curves.

Given two metrics, we consider the $(1,1)$-tensor $L=L(g, \bar{g})$, defined by

$$
L_{j}^{i}:=\left(\frac{\operatorname{det}(\bar{g})}{\operatorname{det}(g)}\right)^{\frac{1}{n+1}} \bar{g}^{i k} g_{k j} .
$$

(Replacing $g$ by $-g$ if necessary, we can always assume that (11) is well-defined.)

The goal of this paper is to give two constructions: gluing and splitting.

- The simplified version of the gluing construction does the following. Consider two manifolds $M_{1}$ and $M_{2}$ with pairs of geodesically equivalent metrics $h_{1} \sim \bar{h}_{1}$ on $M_{1}$ and $h_{2} \sim \bar{h}_{2}$ on $M_{2}$. Assume that the corresponding $(1,1)$-tensor fields $L_{1}=L\left(h_{1}, \bar{h}_{1}\right)$ and $L_{2}=L\left(h_{2}, \bar{h}_{2}\right)$ have no common eigenvalues in the sense that for any two points $x_{1} \in M_{1}, x_{2} \in M_{2}$ we have

$$
\text { Spectrum } L_{1}\left(x_{1}\right) \cap \operatorname{Spectrum} L_{2}\left(x_{2}\right)=\varnothing .
$$

Then one can naturally construct a pair of geodesically equivalent metrics $g \sim \bar{g}$ on the direct product $M=M_{1} \times M_{2}$. These new metrics $g$ and $\bar{g}$ differ from the direct product metrics $h_{1}+h_{2}$ and $\bar{h}_{1}+\bar{h}_{2}$ on $M_{1} \times M_{2}$, but can be obtained from them by explicit formulas involving $L_{1}$ and $L_{2}$ (see (8), (9) below). We denote this by

$$
(M, g, \bar{g})=\left(M_{1}, h_{1}, \bar{h}_{1}\right) \times_{\left(L_{1}, L_{2}\right)}\left(M_{2}, h_{2}, \bar{h}_{2}\right) .
$$

Received by the editors April 16, 2009.

2010 Mathematics Subject Classification. Primary 53A20, 53A35, 53A45, 53B20, 53B30, $53 \mathrm{C} 12,53 \mathrm{C} 21,53 \mathrm{C} 22,37 \mathrm{~J} 35$.

(C)2011 American Mathematical Society Reverts to public domain 28 years from publication 
The corresposponding $(1,1)$ - tensor $L=L(g, \bar{g})$ is, however, the direct sum of $L_{1}$ and $L_{2}$ in the natural sense: for every

$$
\xi=(\underbrace{\xi_{1}}_{\in T_{x_{1}} M_{1}}, \underbrace{\xi_{2}}_{\in T_{x_{2}} M_{2}}) \in T_{\left(x_{1}, x_{2}\right)}\left(M_{1} \times M_{2}\right) \text { we have } L(\xi)=\left(L_{1}\left(\xi_{1}\right), L_{2}\left(\xi_{2}\right)\right) \text {. }
$$

- The splitting construction is the inverse operation. Its local version can be described as follows.

Consider a manifold $M$ with two geodesically equivalent metrics $g \sim \bar{g}$. Assume that at a point $p \in M$ the corresponding $(1,1)$ - tensor $L=L(g, \bar{g})$ has at least two distinct eigenvalues that are not conjugates of each other. Then choose a partition of the spectrum of $L$ into two disjoint nonempty subsets $S_{1} \sqcup S_{2}$ such that each pair of complex-conjugate eigenvalues lies in the same subset. Equivalently, one can say that the characteristic polynomial $\chi(t)$ of $L$ is factorised into two real polynomials $\chi_{1}(t) \cdot \chi_{2}(t)$ with no common roots and $S_{i}$ is just the set of roots of $\chi_{i}(t)$.

Then there is a neighborhood $U=U(p) \subset M$ such that in the above notation the triple $(U, g, \bar{g})$ can be presented as

$$
(U, g, \bar{g})=\left(U_{1}, h_{1}, \bar{h}_{1}\right) \times_{\left(L_{1}, L_{2}\right)}\left(U_{2}, h_{2}, \bar{h}_{2}\right),
$$

with $\operatorname{dim} U_{1}=\operatorname{deg} \chi_{1}, \operatorname{dim} U_{2}=\operatorname{deg} \chi_{2}$. The splitting construction gives an explicit formula for the pair of geodesically equivalent metrics $h_{i} \sim \bar{h}_{i}$ on $U_{i}$ in terms of $g, \bar{g}, L$ and the chosen factorisation $\chi(t)=\chi_{1}(t) \cdot \chi_{2}(t)$ (see (5), (6) below).

The splitting construction is, of course, more important: it allows one to "decompose" $(M, g, \bar{g})$ at least locally into simpler pieces $\left(U_{i}, h_{i}, \bar{h}_{i}\right)$ of the same kind. From the topological viewpoint this decomposition (i.e., the existence of two transversal foliations of complementary dimensions on $M$ ) is, in fact, very natural and is induced by the $(1,1)$-tensor field $L$ itself. We show that the Nijenhuis torsion of $L$ vanishes (Theorem 11) and, as a result, the partition $S_{1} \sqcup S_{2}$ of the spectrum of $L$ leads immediately to two natural integrable distributions that define on $M$ the desired foliations (Theorem 21). The geometric part of the construction is much deeper. We show in particular that $M$ carries two hidden locally product pseudoRiemannian metrics $h$ and $\bar{h}$ which can be canonically reconstructed from $g, \bar{g}$ by means of some nontrivial (and, in our opinion, beautiful) formulas (Theorem 3) involving the information about the partition of the spectrum. Notice that we formulate all of our results in invariant terms which makes it possible to apply them to study global properties of manifolds with geodesically equivalent metrics.

In the Riemannian case, these constructions were obtained in Ma1, Ma5 and played an important role in recent developments in the theory of geodesically equivalent Riemannian metrics. Roughly speaking, these constructions allow one to reduce many natural questions about geodesically equivalent metrics to the case when the $(1,1)$-tensor $L(g, \bar{g})$ has one real eigenvalue, or two complex-conjugate eigenvalues.

We expect similar applications in the theory of pseudo-Riemannian metrics. The main idea is still the same: by iterating the splitting construction, each pair of geodesically equivalent metrics $(g, \bar{g})$ can be canonically "decomposed" into natural "components" $\left(h_{i}, \bar{h}_{i}\right), i=1, \ldots, s$, of smaller dimensions with the same property $h_{i} \sim \bar{h}_{i}$, but with a much simpler structure of the tensor $L_{i}=L\left(h_{i}, \bar{h}_{i}\right)$. Moreover, some other important geometrical objects appearing in this context inherit this 
decomposition. Thus, we are able to work with individual "components" and, as a result, to reduce essentially technical difficulties. We give more details and comments in Sections 2.1, 2.2.

It is worth noticing that compared to Riemannian geometry, the gluing/splitting construction in the pseudo-Riemannian case seems to be even more important because it replaces to some extent the classical Levi-Civita theorem providing a canonical form for a pair of geodesically equivalent Riemannian metrics. The absence of a reasonable analogue of the Levi-Civita theorem in the pseudo-Riemannian case is, in fact, the main reason why the "Riemannian" proof of the gluing/splitting theorems cannot be generalised to the case of pseudo-Riemannian metrics, where one has to develop essentially different techniques (see Remark 6).

A part of our proof of Theorems 3 and 4 could be considered as a separate result. It is a generalisation of a construction by Sinjukov [Si1 and Topalov [To, MT2] which makes it possible to build, starting from a given pair of geodesically equivalent metrics $g \sim \bar{g}$, a whole family of geodesically equivalent pairs $g_{f} \sim \bar{g}_{f}$. We give an invariant explanation of this construction which works both in the Riemannian and pseudo-Riemannian contexts and show that such a family is, in fact, very large: as its parameter, $f$, one can take an arbitrary real-analytic function satisfying certain natural assumptions; see Section 1.3 for more details.

\subsection{Splitting Lemma.}

Definition 2. A local-product structure on $M^{n}$ is a triple $\left(h, B_{1}, B_{2}\right)$, where $h$ is a pseudo-Riemannian metric, and $B_{1}, B_{2}$ are foliations of dimensions $r$ and $n-r$ $(1 \leq r<n)$ such that in a neighborhood of each point $p \in M^{n}$ one can choose local coordinates

$$
(x, y)=\left(\left(x^{1}, x^{2}, \ldots, x^{r}\right),\left(y^{r+1}, y^{r+2}, \ldots, y^{n}\right)\right),
$$

satisfying the following conditions:

1) the leaves of $B_{1}$ are given by $y=$ const $\in \mathbb{R}^{n-r}$;

2) the leaves of $B_{2}$ are given by $x=$ const $\in \mathbb{R}^{r}$;

3) the metric $h$ takes the form

$$
d s_{h}^{2}=\sum_{i, j=1}^{r} h_{i j}(x) d x^{i} d x^{j}+\sum_{\alpha, \beta=r+1}^{n} h_{\alpha \beta}(y) d y^{\alpha} d y^{\beta} ;
$$

in other words, the matrix of $h$ is block-diagonal and its first $r \times r$ block depends on the $x$-coordinates and the second $(n-r) \times(n-r)$ block depends on the $y$-coordinates:

$$
h=\left(\begin{array}{cc}
h_{1}(x) & 0 \\
0 & h_{2}(y)
\end{array}\right) .
$$

Example 1. A model example of manifolds with local-product structure is obviously the direct product of two pseudo-Riemannian manifolds $\left(M_{1}^{r}, h_{1}\right)$ and $\left(M_{2}^{n-r}, h_{2}\right)$. In this case, the leaves of the foliations $B_{1}$ and $B_{2}$ are, respectively, $M_{1}^{r} \times\{y\}, y \in M_{2}^{n-r}$ and $M_{2}^{n-r} \times\{x\}, x \in M_{1}^{r}$. The metric $h$ is the usual product metric $h_{1}+h_{2}$. Locally, every local-product structure is as in this model example.

Geometrically, the existence of a local-product structure for a given metric $h$ is equivalent to the following condition: $T_{p} M$ splits into the direct sum of two nontrivial orthogonal subspaces $U, V \subset T_{p} M$ invariant with respect to the holonomy group. Indeed, if $\left(h, B_{1}, B_{2}\right)$ is a local-product structure, then $U=T_{p} B_{1}$ and $V=T_{p} B_{2}$ are such subspaces. If the orthogonal subspaces $U, V \subset T_{p} M$ (such that 
$\left.U \oplus V=T_{p} M\right)$ are invariant with respect to the holonomy group, then, as was shown by de Rham [DR] and $\mathrm{Wu}[\mathrm{Wu}$, the parallel translation of these subspaces does not depend on the curve and generates integrable distributions, whose integral manifolds form the foliations $B_{1}$ and $B_{2}$ satisfying Definition 2

For two metrics $g$ and $\bar{g}$, we consider the $(1,1)$-tensor $L=L(g, \bar{g})$ given by (1). Its characteristic polynomial will be denoted by

$$
\chi: M \times \mathbb{R} \rightarrow \mathbb{R}, \quad \chi(t):=\operatorname{det}(t \cdot \mathrm{Id}-L) .
$$

It is a polynomial of degree $n$ whose coefficients are smooth functions on the manifold. Note that by our definition the polynomial is monic, i.e., its leading coefficient is 1 .

We say that a factorisation of $\chi(t)$ into two monic real polynomials

$$
\chi(t)=\chi_{1}(t) \cdot \chi_{2}(t), \quad \operatorname{deg} \chi_{i} \geq 1, \quad \chi_{i}: M \times \mathbb{R} \rightarrow \mathbb{R}
$$

is admissible if $\chi_{1}(t)$ and $\chi_{2}(t)$ are coprime (i.e., have no common roots) at every point $x \in M$.

Example 2. Let $\lambda_{1}, \ldots, \lambda_{n}$ be (possibly, complex) eigenvalues of $L$ at a point $p \in M$ counted with their algebraic multiplicities. Divide them into two nonempty groups (without loss of generality we think that $\lambda_{1}, \ldots, \lambda_{r}$ lie in the first group and $\lambda_{r+1}, \ldots, \lambda_{n}$ in the second group) in such a way that $\lambda_{i} \neq \lambda_{\alpha}$ for $i=1, \ldots, r$, $\alpha=r+1, \ldots, n$, and pairs of complex-conjugate eigenvalues lie in the same group. Then, the polynomials

$$
\chi_{1}(t):=\left(t-\lambda_{1}\right) \ldots\left(t-\lambda_{r}\right), \quad \chi_{2}(t):=\left(t-\lambda_{r+1}\right) \ldots\left(t-\lambda_{n}\right)
$$

are real and give an admissible factorisation of $\chi(t)$ at $p \in M$. By using the implicit function theorem, it is easy to see that this factorisation can always be extended onto some neighborhood $U(p) \in M$.

Locally every admissible factorisation is as in this example. However, the existence of a local admissible factorisation at every point does not imply its global existence.

Given an admissible factorisation $\chi=\chi_{1} \cdot \chi_{2}$, we consider the following two distributions $D_{1}, D_{2}$ on $M$ :

$$
D_{i}=\operatorname{ker} \chi_{i}(L), \quad i=1,2,
$$

where ker denotes the kernel. Here (and in all other places of the paper where we consider polynomials in $L$ with coefficients being smooth functions on $M$ ) we treat the $(1,1)$-tensor $L$ as a linear operator acting on each tangent space, and a polynomial $f(L)$ in $L$ is the $(1,1)$-tensor of the form $f(L)=a_{0}(x) \cdot \operatorname{Id}+a_{1}(x) L+$ $a_{2}(x) L^{2}+\cdots+a_{m}(x) L^{m}$.

It is easy to see that $D_{1}$ and $D_{2}$ are transversal distributions of complementary dimensions. Moreover, the distributions are invariant with respect to $L$ and are mutually orthogonal with respect to both metrics $g$ and $\bar{g}$.

Theorem 1. Let $g$ and $\bar{g}$ be geodesically equivalent pseudo-Riemannian metrics on $M, L=L(g, \bar{g})$ be the $(1,1)$-tensor associated with them, and $\chi=\chi_{1} \cdot \chi_{2}$ be an admissible factorisation of its characteristic polynomial. Then, the distributions (3) are integrable. 
By Theorem 11, the admissible factorisation implies the (local) existence of a coordinate system $\left(x^{1}, \ldots, x^{r}, y^{r+1}, \ldots, y^{n}\right)$ such that the distribution $D_{1}$ is generated by the coordinate vector fields $\partial_{x^{i}}, i=1, \ldots, r$, and similarly $D_{2}$ is generated by $\partial_{y^{j}}, j=r+1, \ldots, n$. Since the distributions are invariant with respect to $L$, in this coordinate system the matrix of $L$ is block-diagonal:

$$
L(x, y)=\left(\begin{array}{cc}
L_{1}(x, y) & 0 \\
0 & L_{2}(x, y)
\end{array}\right)
$$

where $L_{1}$ and $L_{2}$ are $r \times r-$ and $(n-r) \times(n-r)-$ matrices, respectively.

Theorem 2. Under the assumptions of Theorem 1 and in the notation above, the entries of $L_{1}$ depend on the $x$-variables only, and the entries of $L_{2}$ depend on the $y$-variables only, so

$$
L(x, y)=\left(\begin{array}{cc}
L_{1}(x) & 0 \\
0 & L_{2}(y)
\end{array}\right) .
$$

By Theorem 1, the distributions $D_{1}$ and $D_{2}$ generate two foliations on the manifold. We denote them by $B_{1}$ and $B_{2}$, respectively.

Theorem 3 (Splitting Lemma). Let $g$ and $\bar{g}$ be geodesically equivalent pseudoRiemannian metrics on $M$, and let $B_{1}, B_{2}$ be the foliations generated by the distributions (3) related to an admissible factorisation $\chi=\chi_{1} \cdot \chi_{2}$ of the characteristic polynomial of $L=L(g, \bar{g})$. Then, the following two $(0,2)$-tensors

$$
\begin{aligned}
& h_{i j}=g_{i k}\left(\left(\chi_{2}(L)+\chi_{1}(L)\right)^{-1}\right)_{j}^{k} \\
& \bar{h}_{i j}=\bar{g}_{i k}\left(\left(\frac{1}{\chi_{2}(0)} \chi_{2}(L)+\frac{1}{\chi_{1}(0)} \chi_{1}(L)\right)^{-1}\right)_{j}^{k}
\end{aligned}
$$

are pseudo-Riemannian metrics (i.e., symmetric and nondegenerate), and the triples $\left(h, B_{1}, B_{2}\right)$ and $\left(\bar{h}, B_{1}, B_{2}\right)$ are local-product structures on $M$. Moreover, for every leaf $M_{i}$ of the foliation $B_{i}$, the restrictions $h_{i}=\left.h\right|_{M_{i}}$ and $\left.\bar{h}_{i}\right|_{M_{i}}$ of the metrics $h$ and $\bar{h}$ to this leaf are geodesically equivalent, and the tensor $L_{i}=L\left(h_{i}, \bar{h}_{i}\right)$ defined on $M_{i}$ by (11) coincides with the restriction $\left.L\right|_{M_{i}}$ of $L$ to $M_{i}, i=1,2$. Moreover, $\chi_{1}=\chi_{L_{1}}$ and $\chi_{2}=\chi_{L_{2}}$.

In formula (6), the expression $\chi_{i}(0), i=1,2$, denotes the smooth function on $M$ obtained by substituting $t=0$ into $\chi_{i}(t)$, namely, $\chi_{1}(0)=(-1)^{r} \operatorname{det} L_{1}$, and $\chi_{2}(0)=(-1)^{n-r} \operatorname{det} L_{2}$.

Remark 1. It might be convenient to understand the formulas (5), (6) in matrix notation: let us consider the coordinate system $\left(x^{1}, \ldots, x^{r}, y^{r+1}, \ldots, y^{n}\right)$ such that the distribution $D_{1}$ is generated by the vectors $\partial_{x^{i}}$, and the distribution $D_{2}$ is generated by the vectors $\partial_{y^{j}}$. Then, in this coordianate system all matrices we use to construct $h$ and $\bar{h}$ are block-diagonal with one $r \times r-$ and one $(n-r) \times(n-r)$-block:

$$
g=\left(\begin{array}{cc}
g_{1} & 0 \\
0 & g_{2}
\end{array}\right), \quad \bar{g}=\left(\begin{array}{cc}
\bar{g}_{1} & 0 \\
0 & \bar{g}_{2}
\end{array}\right), \quad L=\left(\begin{array}{cc}
L_{1} & 0 \\
0 & L_{2}
\end{array}\right)
$$


Hence, the matrices of $h$ and $\bar{h}$ must be block-diagonal as well, and direct calculations give us the formulas

$$
\begin{aligned}
& h=\left(\begin{array}{cc}
g_{1} \chi_{2}\left(L_{1}\right)^{-1} & 0 \\
0 & g_{2} \chi_{1}\left(L_{2}\right)^{-1}
\end{array}\right), \\
& \bar{h}=\left(\begin{array}{cc}
\chi_{2}(0) \bar{g}_{1} \chi_{2}\left(L_{1}\right)^{-1} & 0 \\
0 & \chi_{1}(0) \bar{g}_{2} \chi_{1}\left(L_{2}\right)^{-1}
\end{array}\right) .
\end{aligned}
$$

Remark 2. Although the geodesic equivalence relation is obviously symmetric, the tensor $L$ is not invariant with respect to the permutation of $g$ and $\bar{g}$. More precisely, the tensor (1) constructed for $\bar{g}, g$ is the inverse of (1) constructed for $g, \bar{g}$. Moreover, two local product structures constructed from the pair of geodesically equivalent metrics $g, \bar{g}$ do not coincide with the local product structure constructed from the pair $\bar{g}, g$, though of course they are closely related; in particular (after the proper choice of admissible factorisations) all these four local-product structures share the same foliations $B_{1}$ and $B_{2}$.

1.2. Gluing Lemma. Let $B_{1}$ and $B_{2}$ be two transversal foliations of complementary dimensions, and let $h, \bar{h}$ be two metrics such that the triples $\left(h, B_{1}, B_{2}\right)$ and $\left(\bar{h}, B_{1}, B_{2}\right)$ are local-product structures.

The tangent spaces to the leaves of the foliations will be denoted by $T B_{1}, T B_{2}$, and for every $p \in M$ we have $T_{p} M=T_{p} B_{1} \oplus T_{p} B_{2}$.

We denote by $h_{i}, \bar{h}_{i}$ the restrictions of the metrics $h, \bar{h}$ to the leaves of $B_{i}, i=$ 1,2. The corresponding tensor (11) will be denoted by $L_{i}:=L\left(h_{i}, \bar{h}_{i}\right)$, and its characteristic polynomial by $\chi_{i}, i=1,2$. Assume in addition that the polynomials $\chi_{1}$ and $\chi_{2}$ are coprime for all points of $M$.

Now, consider the following two symmetric bilinear forms $g, \bar{g}$ on $M$ : for two tangent vectors

$$
u=(\underbrace{u_{1}}_{\in T B_{1}}, \underbrace{u_{2}}_{\in T B_{2}}), \quad v=(\underbrace{v_{1}}_{\in T B_{1}}, \underbrace{v_{2}}_{\in T B_{2}}) \in T M
$$

we put

$$
\begin{aligned}
& g(u, v)=h_{1}\left(\chi_{2}\left(L_{1}\right)\left(u_{1}\right), v_{1}\right)+h_{2}\left(\chi_{1}\left(L_{2}\right)\left(u_{2}\right), v_{2}\right), \\
& \bar{g}(u, v)=\frac{1}{\chi_{2}(0)} \bar{h}_{1}\left(\chi_{2}\left(L_{1}\right)\left(u_{1}\right), v_{1}\right)+\frac{1}{\chi_{1}(0)} \bar{h}_{2}\left(\chi_{1}\left(L_{2}\right)\left(u_{2}\right), v_{2}\right) .
\end{aligned}
$$

Theorem 4 (Gluing Lemma). If $h_{1}$ is geodesically equivalent to $\bar{h}_{1}$, and $h_{2}$ is geodesically equivalent to $\bar{h}_{2}$, then the metrics $g, \bar{g}$ given by (8), (9) are geodesically equivalent too.

Remark 3. It is an easy linear algebra to see that the tensor (1) constructed for the metrics (8), (9) is the direct sum of the tensors $L_{1}$ and $L_{2}$ : for every $u=\left(u_{1}, u_{2}\right) \in$ $T_{p} B_{1} \oplus T_{p} B_{2}=T_{p} M$ we have

$$
L(u)=\left(L_{1}\left(u_{1}\right), L_{2}\left(u_{2}\right)\right) .
$$

Remark 4. It might again be convenient to understand the formulas (8), (9) in matrix notation: we consider the coordinate system $\left(x^{1}, \ldots, x^{r}, y^{r+1}, \ldots, y^{n}\right)$ such that the $y$-coordinates are constant on the leaves of $B_{1}$ and the $x$-coordinates are constant on the leaves of $B_{2}$. Then, in this coordinate system, the matrices of $g$ 
and $\bar{g}$ are given by

$$
g=\left(\begin{array}{cc}
h_{1} \chi_{2}\left(L_{1}\right) & 0 \\
0 & h_{2} \chi_{1}\left(L_{2}\right)
\end{array}\right), \quad \bar{g}=\left(\begin{array}{cc}
\frac{1}{\chi_{2}(0)} \bar{h}_{1} \chi_{2}\left(L_{1}\right) & 0 \\
0 & \frac{1}{\chi_{1}(0)} \bar{h}_{2} \chi_{1}\left(L_{2}\right)
\end{array}\right) .
$$

Remark 5. Comparing formulas (77) and (11), we see that the gluing construction is inverse to the splitting.

Remark 6 . In the case of Riemannian metrics, Theorems 3 and 4 were proven in Ma1, Ma5. The proof is based on the Levi-Civita description of geodesically equivalent metrics, which is not complete in the pseudo-Riemannian case; see the discussion in Section 2.1. Below, we will show that the Levi-Civita description is an easy corollary of Theorems 3 and 4 , see the example in Section 2.1.

1.3. Functions of $(1,1)$-tensors and the Topalov-Sinjukov Theorem for pseudo-Riemannian metrics. Suppose a compact set $K \subseteq \mathbb{C}$, a function $f$ : $K \rightarrow \mathbb{C}$, and a $(1,1)$-tensor $L$ (on $M$ ) satisfy the following assumptions:

(i) $\mathbb{C} \backslash K$ is connected (we do not require that $K$ is connected).

(ii) $f: K \rightarrow \mathbb{C}$ is a continuous function, and the restriction $\left.f\right|_{\operatorname{Int} K}$ is holomorphic.

(iii) $K$ is symmetric with respect to the $x$-axes: for every $z \in K$ its conjugate $\bar{z}$ also lies in $K$.

(iv) For every $z \in K, f(z)=\bar{f}(\bar{z})$, where the bar "-" denotes complex conjugation.

(v) Spectrum $L(x) \subset \operatorname{Int} K$ for every $x \in M$.

Under the above assumptions (i) $-(\mathbb{V})$, one can naturally define a $(1,1)$-tensor $f(L)$ : by Mergelyan's theorem [Ca], the function $f$ can be uniformly approximated by real polynomials $p_{i}$. We define $f(L)=\lim _{i \rightarrow \infty} p_{i}(L)$. It is an easy exercise (see for example [Hi, §1.2.2-1.2.4]) to show that the limit exists, is independent of the choice of the sequence $p_{i}$, smoothly depends on $x \in M^{m}$ (actually, the function $f(L)$ is analytic in the entries of $L)$, and behaves as a $(1,1)$-tensor when we change the variables.

Example 3. Polynomials $p(z)$ with real coefficients, the functions $e^{z}, \cos (z), \sin (z)$ satisfy the above assumptions for every $L$, and we can naturally consider the standard operator functions $p(L), e^{L}, \sin (L), \cos (L)$ as smooth $(1,1)$-tensor fields on $M$.

Example 4. Let $\chi=\chi_{1} \cdot \chi_{2}$ be an admissible factorisation of the characteristic polynomial of $L$ in a sufficiently small neighborhood $U\left(x_{0}\right) \subset M$ and let $D_{1}, D_{2}$ be the corresponding distributions. Then, the natural projectors $P_{i}: T U \rightarrow D_{i}$ are functions of $L$ in the above sense. Indeed, consider the corresponding partition $S_{1} \sqcup S_{2}$ of the Spectrum $L\left(x_{0}\right)$ into two disjoint subsets and let $K_{1}$ and $K_{2}$ be $\varepsilon$ neighborhoods of $S_{1}$ and $S_{2}$, respectively ( $\varepsilon$ is small enough so that $K_{1}$ and $K_{2}$ do not intersect). Then, the function

$$
f_{1}: K \rightarrow \mathbb{C}, \quad f_{1}(z):= \begin{cases}1 & \text { for } z \in K_{1} \\ 0 & \text { for } z \in K_{2}\end{cases}
$$

satisfies the above assumptions, and we can define the function $f_{1}(L)$ which obviously coincides with the projector $P_{1}$. Similarly, one can define $f_{2}(L)=P_{2}$.

Notice that for every $x \in M$ we have: $\operatorname{Spectrum} f(L(x))=f(\operatorname{Spectrum} L(x))$. In particular, if for every $x \in M$ we have $0 \notin f$ (Spectrum $L(x))$, then the tensor $f(L)$ 
is nondegenerate. If in addition $L$ is $g$-selfadjoint, then $f(L)$ is also $g$-selfadjoint, so $g_{f}:=\left(g_{i \alpha} f(L)_{j}^{\alpha}\right)$ is a metric.

Theorem 5. Let $g$ and $\bar{g}$ be geodesically equivalent metrics and let $L$ be the $(1,1)-$ tensor given by (11). Suppose $K \subset \mathbb{C}$ is a compact set, and $f: K \rightarrow \mathbb{C}$ is a function such that $K, f$, and $L$ satisfy the assumptions (ii) -(ㅈ) ) above. Assume in addition that for every $x \in M$ we have $0 \notin f$ (Spectrum $L(x))$.

Then, the metrics $g_{f}:=\left(g_{i \alpha} f(L)_{j}^{\alpha}\right)$ and $\bar{g}_{f}:=\left(\bar{g}_{i \alpha} f(L)_{j}^{\alpha}\right)$ are also geodesically equivalent.

Remark 7. Partial cases of this theorem were proved by Sinjukov [Si1] and Topalov To, MT2. More precisely, Sinjukov proved this theorem assuming that the function $f(z)=z$. Topalov proved the theorem assuming the metric $g$ is Riemannian (in this case the eigenvalues of $L$ are real, and is sufficient to require that the function $f$ is real-analytic).

\section{History, motivation AND POSSIBLE APPLiCATiOns}

The theory of geodesically equivalent metrics has a long and fascinating history. The first nontrivial examples were discovered by Lagrange [La]. Geodesically equivalent metrics were studied by Beltrami [Be, Levi-Civita [LC], Painlevé [Pa] and other classics. One can find more historical details in the surveys [Am2, Mi2] and in the introductions to the papers [Ma1, Ma2, Ma4, Ma5].

The success of general relativity made necessary the study of geodesically equivalent pseudo-Riemannian metrics. The textbooks [Ei, Pe1, Pe2, Hal3] on pseudoRiemannian metrics have chapters on geodesically equivalent metrics. In the popular paper We2, Weyl stated a few interesting open problems on geodesic equivalence of pseudo-Riemannian metrics. Recent references (on the connection between geodesically equivalent metrics and general relativity) include Hall et al. HL1, HL2, HL3, Hall [Hal1, Hal2, Kiosak et al. [KM1, and Gibbons et al. GWW.

Recently, huge progress was made in the theory of geodesically equivalent Riemannian metrics. The splitting/gluing constructions played a crucial role in this progress (as we mentioned in Remark 6, in the Riemannian case, Theorems 3 and 4 were known). We expect similar applications in the pseudo-Riemannian situation as well. The list of problems where the splitting/gluing constructions were used in the Riemannian case, and are expected to be used in the pseudo-Riemannian case, is below; we discuss it in detail in Sections 2.1, 2.2,

Beltrami Problem 1 Describe all pairs of geodesically equivalent metrics.

Lie Problem 2 Find all metrics $g$ admitting infinitesimal projective transformations.

Lichnerowicz Conjecture 3 Let a connected Lie group $G$ act on a complete connected manifold $\left(M^{n}, g\right)$ of dimension $n \geq 2$ by projective transformations.

\footnotetext{
${ }^{1}$ Italian original from $[\mathrm{Be}$ : La seconda ... generalizzazione ... del nostro problema, vale a dire: riportare $i$ punti di una superficie sopra un'altra superficie in modo che alle linee geodetiche della prima corrispondano linee geodetiche della seconda.

${ }^{2}$ German original from [Lie], Abschn. I, Nr. 4, Man soll die Form des Bogenelementes einer jeden Fläche bestimmen, deren geodätische Kurven eine infinitesimale Transformation gestatten.

${ }^{3}$ The attribution to Lichnerowicz is folkloric. We did not find a paper by Lichnerowicz where he states this conjecture. Certain papers refer to this statement as to a classical conjecture; see the discussion in $\mathrm{Ma5}$.
} 
Then, it acts by affine transformations, or for some $c \in \mathbb{R} \backslash\{0\}$ the metric $c \cdot g$ is the Riemannian metric of constant positive sectional curvature +1 .

Recall that a projective transformation of a Riemannian manifold is a diffeomorphism of the manifold that takes unparameterized geodesics to geodesics. Local projective transformations obviously form a Lie pseudo-group. Its generators, i.e., vector fields whose local flow takes unparameterized geodesics to geodesics, are called by Lie infinitesimal projective transformations. In the modern terminlogy, they are called projective vector fields; we will use this terminology in the paper.

2.1. Motivation I. Normal form of geodesically equivalent metrics: Beltrami problem. If the eigenvalues of $L$ do not bifurcate at a point (this condition is fulfilled almost everywhere), the answer to Beltrami's question was given by Levi-Civita [LC under the assumption that the metrics are Riemannian.

A local description of geodesically equivalent pseudo-Riemannain metrics, which might be treated as a pseudo-Riemannian analog of the Levi-Civita theorem, is considered to have been done by Aminova Am1. Unfortunately, the authors of the present paper do not understand her result (we do not doubt that the result is correct). Moreover, we have checked that in all 10 papers which refer to Am1] according to MathSciNet, the authors cited Aminova's theorem to give an overview of the subject only, but did not really use it.

The statement of the main theorem of [Am1] is on two pages, and one more page is devoted to an explanation of the formulas in the theorem. This is probably the reason why this result is hardly applicable and was, to the best of our knowledge, never used.

The splitting and gluing constructions are supposed to make the description of geodesically equivalent pseudo-Riemannian metrics much simpler. Indeed, at almost every point $p \in M$ the eigenvalues of $L$ do not bifurcate; i.e., the algebraic multiplicity of each eigenvalue $\lambda_{i}$ is locally constant and is equal to $k_{i}$. In a small neighborhood of such a point, the eigenvalues (both real and complex) can be treated as smooth functions of $x$, and we can factorise the characteristic polynomial of $L$ into a product $\chi=\chi_{1} \cdot \ldots \cdot \chi_{m}$ of polynomials $\chi_{i}: \mathbb{R} \times M \rightarrow \mathbb{R}$ of two kinds: either $\chi_{i}(t)=\left(t-\lambda_{i}\right)^{k_{i}}$ for a real eigenvalue $\lambda_{i}$, or $\chi_{i}(t)=\left(t-\lambda_{i}\right)^{k_{i}}\left(t-\bar{\lambda}_{i}\right)^{k_{i}}$ for a pair of complex conjugate eigenvalues $\lambda_{i}, \bar{\lambda}_{i}$.

Repeatedly applying the splitting lemma $m-1$ times in a small neighborhood $U(p)$, we can construct local coordinates $x_{1}, \ldots, x_{m}$, where $x_{i}=\left(x_{i}^{1}, \ldots, x_{i}^{l_{i}}\right) \in \mathbb{R}^{l_{i}}$ ( $l_{i}=k_{i}$ if $\lambda_{i}$ is real, and $l_{i}=2 k_{i}$ for a couple of complex conjugate eigenvalues $\left.\lambda_{i}, \bar{\lambda}_{i}\right)$, and pairs of geodesically equivalent metrics $h_{i}\left(x_{i}\right) \sim \bar{h}_{i}\left(x_{i}\right)$ such that

1) each eigenvalue $\lambda_{i}$ of $L$ depends on $x_{i}$ only: $\lambda_{i}=\lambda_{i}\left(x_{i}\right)$;

2) the characteristic polynomial of the $(1,1)$-tensor $L_{i}=L\left(h_{i}, \bar{h}_{i}\right)$ associated with $h_{i}$ and $\bar{h}_{i}$ by means of (1) is exactly $\chi_{i}(t)$;

3 ) in this coordinate system, $g$ and $\bar{g}$ simultaneously take the following block diagonal form:

$$
g=\left(\begin{array}{ccc}
h_{1} \hat{\chi}_{1}\left(L_{1}\right) & & \\
& \ddots & \\
& & h_{m} \hat{\chi}_{m}\left(L_{m}\right)
\end{array}\right), \quad \bar{g}=\left(\begin{array}{lll}
\frac{1}{\hat{\chi}_{1}(0)} \bar{h}_{1} \hat{\chi}_{1}\left(L_{1}\right) & & \\
& & \ddots \\
& & \frac{1}{\hat{\chi}_{m}(0)} \bar{h}_{m} \hat{\chi}_{m}\left(L_{m}\right)
\end{array}\right),
$$

where $\hat{\chi}_{i}(t)=\prod_{j \neq i} \chi_{j}(t)$. 
Thus we have

Proposition 1. In a neighborhood of a regular point $p \in M$, the geodesically equivalent metrics $g$ and $\bar{g}$ can be simultaneously reduced by an appropriate choice of local coordinates $x_{1}, \ldots, x_{m}, x_{i} \in \mathbb{R}^{l_{i}}$, to the form (12), where $h_{i}$ and $\bar{h}_{i}$ are geodesically equivalent metrics depending on $x_{i}$-coordinates only and such that the corresponding $(1,1)$-tensor $L_{i}=L\left(h_{i}, \bar{h}_{i}\right)$ has either one single real eigenvalue $\lambda_{i}\left(x_{i}\right)$, or a single pair of complex conjugate eigenvalues $\lambda_{i}\left(x_{i}\right), \bar{\lambda}_{i}\left(x_{i}\right)$.

Thus, in order to describe geodesically equivalent metrics near a generic point it is sufficient to do this under the assumption that $L$ has one real eigenvalue, or two complex-conjugate eigenvalues. In the Riemannian case, we will illustrate this idea by the following.

Example (Levi-Civita Theorem follows from the Splitting Lemma). Let the geodesically equivalent metrics $g \sim \bar{g}$ be Riemannian. Then all eigenvalues of $L$ are real and positive, and $L$ is semi-simple. Hence, the tensor $L\left(h_{i}, \bar{h}_{i}\right)$ for geodesically equivalent metrics $h_{i} \sim \bar{h}_{i}$ discussed above is $\lambda_{i} \cdot$ Id. If the multiplicity $k_{i}$ of $\lambda_{i}$ is $\geq 2$, then by the classical result of Weyl We1 the metrics $h_{i}$ and $\bar{h}_{i}$ are proportional, i.e., $\bar{h}_{i}:=\frac{1}{\lambda_{i}^{k_{i}+1}} h_{i}$, where the eigenvalue $\lambda_{i}$ is a constant.

If the multiplicity $k_{i}$ of $\lambda_{i}$ is 1 , then $h_{i}$ is one-dimensional and we can obviously choose $x_{i}$ in such a way that $h_{i}=d x_{i}^{2}$ and $\bar{h}_{i}=\frac{1}{\lambda_{i}\left(x_{i}\right)^{2}} d x_{i}^{2}$.

Without loss of generality we can assume that the first $r$ eigenvalues $\lambda_{1}, \ldots, \lambda_{r}$ have multiplicity 1 , and the last $m-r$ eigenvalues have multiplicity $\geq 2$. Then, in the chosen coordinate system, the direct product metrics $h=h_{1}+h_{2}+\cdots+h_{m}$ and $\bar{h}=\bar{h}_{1}+\bar{h}_{2}+\cdots+\bar{h}_{m}$ are given by

$$
\begin{aligned}
d s_{h}^{2} & =\sum_{i=1}^{r} d x_{i}{ }^{2}+\sum_{i=r+1}^{m}\left[\sum_{\alpha_{i}, \beta_{i}=1}^{k_{i}}\left(h_{i}\left(x_{i}\right)\right)_{\alpha_{i} \beta_{i}} d x_{i}^{\alpha_{i}} d x_{i}^{\beta_{i}}\right] \\
d s_{\bar{h}}^{2} & =\sum_{i=1}^{r} \frac{1}{\lambda_{i}\left(x_{i}\right)^{2}} d x_{i}{ }^{2}+\sum_{i=r+1}^{m}\left[\frac{1}{\lambda_{i}^{k+1}} \sum_{\alpha_{i}, \beta_{i}=1}^{k_{i}}\left(h_{i}\left(x_{i}\right)\right)_{\alpha_{i} \beta_{i}} d x_{i}^{\alpha_{i}} d x_{i}^{\beta_{i}}\right] .
\end{aligned}
$$

Here the functions $\lambda_{i}$ are constant for $i>r$ and depend only on the corresponding variable $x_{i}$ for $i \leq r$. The metrics $h_{i}, i=r+1, \ldots, m$ can be arbitrary, but their entries $\left(h_{i}\right)_{\alpha_{i} \beta_{i}}$ must depend on the coordinates $x_{i}=\left(x_{i}^{1}, \ldots, x_{i}^{k_{i}}\right)$ only. form:

Applying Proposition 1 and formula (12), we obtain for $g$ and $\bar{g}$ the following

$$
\begin{aligned}
d s_{g}^{2} & =\sum_{i=1}^{r} P_{i} d x_{i}{ }^{2}+\sum_{i=r+1}^{m}\left[P_{i} \sum_{\alpha_{i}, \beta_{i}=1}^{k_{i}}\left(h_{i}\left(x_{i}\right)\right)_{\alpha_{i} \beta_{i}} d x_{i}^{\alpha_{i}} d x_{i}^{\beta_{i}}\right], \\
d s_{\bar{g}}^{2} & =\sum_{i=1}^{r} P_{i} \rho_{i} d x_{i}{ }^{2}+\sum_{i=r+1}^{m}\left[P_{i} \rho_{i} \sum_{\alpha_{i}, \beta_{i}=1}^{k_{i}}\left(h_{i}\left(x_{i}\right)\right)_{\alpha_{i} \beta_{i}} d x_{i}^{\alpha_{i}} d x_{i}^{\beta_{i}}\right],
\end{aligned}
$$

where

$$
P_{i}:= \pm \prod_{j \neq i}\left(\lambda_{i}-\lambda_{j}\right), \quad \rho_{i}:= \pm \frac{1}{\lambda_{i} \prod_{\alpha} \lambda_{\alpha}}
$$


(the signs \pm should be chosen so that all $P_{i}$ and $\rho_{i}$ are positive). This is precisely the Levi-Civita normal form from [LC] for geodesically equivalent Riemannian metrics!

As we mentioned above, the results by Levi-Civita and Aminova hold in a neighborhood of almost every point. More precisely, such a point $p \in M$, which we call regular, is characterised by the property that the structure of the Jordan normal form of $L$ (the number of Jordan blocks and their dimensions) is the same for all points in some neighborhood $U(p)$. Regular points form an open everywhere dense subset of $M$. The other points will be called singular.

For global questions (in particular, for the description of the topology of closed manifolds admitting geodesically equivalent metrics), it is also necessary to solve the Beltrami problem near singular points. For the Riemannian case, this was done in Ma1, Ma5, Ma6. The result essentially used the splitting construction: arguing as above, the problem was reduced to a few cases of simple bifurcations, which were considered separately. We expect the same application of the splitting construction in the pseudo-Riemannian case.

2.2. Motivation II. Projective transformations: Lie Problem and Lichnerowicz Conjecture. For Riemannian manifolds, the splitting construction found an important application in the theory of projective transformations. The topic of projective transformations is a very classical object of study. The first examples are due to Beltrami [Be]; as we mentioned at the beginning of Section 2 , the problem of local description of projective transformations was explicitly stated by Lie [Lie. In the global setting, namely under the assumption that $(M, g)$ is complete, a hypothetical solution of this problem is the Lichnerowicz Conjecture mentioned above.

In this section we assume that $\operatorname{dim} M \geq 3$. The reason for this is that in dimension 2 the Lie problem was solved in [BMM], [Ma7, where a complete list of local metrics admitting projective vector fields was constructed. The list is pretty simple (explicit formulas involving only elementary functions), and it should not be very complicated to understand which metrics from this list can be prolonged up to a complete metric.

As was observed by Fubini and Solodovnikov, in dimension $\geq 3$, the degree of mobility plays a crucial role in the description of projective transformations of a given manifold. Recall that the degree of mobility of a metric $g$ is the dimension of the space of the solutions of the equation (21) considered as an equation on $L$. The degree of mobility has a clear geometrical meaning: locally, it coincides with the dimension of the set of metrics geodesically equivalent to $g$ equipped with the natural topology.

In particular, the condition "degree of mobility $=1$ " means that $g$ does not admit any geodesically equivalent "partner" except for $\bar{g}=$ const $\cdot g$. In this case projective transformations are just homotheties of $g$.

Riemannian metrics (on $M^{n \geq 3}$ ) with degree of mobility greater than or equal to three and their projective vector fields were locally described by Solodovnikov [So]; his result was improved by Shandra $\underline{\mathrm{Sh}}$, who obtained a much shorter description based on a certain trick. The results of [KM2] show that

- the metrics with degree of mobility $\geq 3$ always admit projective vector fields, 
- the trick used in [Sh] survives for pseudo-Riemannian metrics as well, and gives a description of all metrics with degree of mobility $\geq 3$, and their projective vector fields.

Thus, in order to get the complete solution of the Lie Problem in dimension $\geq 3$, it is sufficient to consider metrics with degree of mobility 2 . It turns out that under this assumption, the problem can be reduced to the analysis of a certain first-order system of PDEs. This system is universal in the sense that it does not depend on the metric and remains the same if we pass from the whole manifold $M$ to the components of smaller dimensions obtained by means of the splitting lemma. We describe this idea in brief below.

First of all we notice that in all our considerations a pair $g \sim \bar{g}$ of geodesically equivalent metrics can be replaced by the pair $(g, L)$, where $L$ is the $(1,1)$-tensor defined by (11). Indeed, $\bar{g}$ can be uniquely reconstructed from $g$ and $L$ as $\bar{g}=$ $\frac{1}{\operatorname{det}(L)} g L^{-1}$. We shall say that $L$ and $g$ are compatible if $g$ and $\bar{g}=\frac{1}{\operatorname{det}(L)} g L^{-1}$ are geodesically equivalent. The $(1,1)$-tensors $L$ compatible with $g$ form a finitedimensional vector space whose dimension is exactly the degree of mobility of $g$.

Now let $v$ be a projective vector field for the metric $g$ (we work in a small connected neighborhood $U$ of a point $p \in M$ ). Consider the tensor

$$
\tilde{L}:=g^{-1} \mathcal{L}_{v} g-\frac{1}{n+1} \operatorname{tr}\left(g^{-1} \mathcal{L}_{v} g\right) \cdot \mathrm{Id},
$$

where $\mathcal{L}_{v}$ denotes the Lie derivative with respect to $v$.

Lemma 1 ([Ma5]). The tensor $\tilde{L}$ given by (14) is compatible with $g$.

Lemma 1 is an easy corollary of the compatibility condition (21) below. Its proof can be found for example in [Ma5, Section 2.1]; see Theorem 3 there. In an equivalent form the statement had already appeared in Fubini [Fu (for dimension 3) and Solodovnikov [So] (under additional assumptions).

Now assume that the degree of mobility of $g$ is 2 , so that the vector space of admissible $(1,1)$-tensors is two-dimensional. Clearly, the identity tensor Id is admissible and we can take it as the first basis vector. We choose the second one, denoted by $L$, in such a way that $\operatorname{det} L \neq 0$ in some neighborhood of $p \in M$.

Lemma 2. Under the above assumptions, the tensor $L$ and the projective vector field $v$ satisfy the following equation:

$$
\mathcal{L}_{v} L=\alpha \cdot L^{2}+\beta \cdot L+\gamma \cdot \mathrm{Id},
$$

where $\beta$ is a function given by $\beta:=-\mathcal{L}_{v}(\log |\operatorname{det} L|) \cdot L+\alpha \cdot \operatorname{tr}(L)+\gamma \cdot \operatorname{tr}\left(L^{-1}\right)+\nu$, and $\alpha, \beta, \nu$ are some constants.

Proof. Consider the metric $\bar{g}=\frac{1}{\operatorname{det}(L)} g L^{-1}$. This metric is geodesically equivalent to $g$, and therefore $v$ is a projective vector field also for $\bar{g}$. Clearly, the degree of mobility of $\bar{g}$ coincides with that of $g$. Hence, the space of $\bar{g}$-compatible $(1,1)-$ tensors is two-dimensional and is generated by Id and $L^{-1}$.

Thus, applying Lemma 1 to $g$ and $\bar{g}$, we have:

$$
\begin{aligned}
& g^{-1} \mathcal{L}_{v} g-\frac{1}{n+1} \operatorname{tr}\left(g^{-1} \mathcal{L}_{v} g\right)=a \cdot L+b \cdot \mathrm{Id}, \\
& \bar{g}^{-1} \mathcal{L}_{v} \bar{g}-\frac{1}{n+1} \operatorname{tr}\left(\bar{g}^{-1} \mathcal{L}_{v} \bar{g}\right)=c \cdot L^{-1}+d \cdot \mathrm{Id},
\end{aligned}
$$


where $a, b, c, d \in \mathbb{R}$ are some constants. It is easy to see that these equations can be rewritten in the following equivalent form:

$$
\begin{aligned}
& g^{-1} \mathcal{L}_{v} g=a \cdot L+(a \cdot \operatorname{tr}(L)+(n+1) b) \cdot \mathrm{Id}, \\
& \bar{g}^{-1} \mathcal{L}_{v} \bar{g}=c \cdot L^{-1}+\left(c \cdot \operatorname{tr}\left(L^{-1}\right)+(n+1) d\right) \cdot \mathrm{Id} .
\end{aligned}
$$

On the other hand, we have

$$
\begin{aligned}
\bar{g}^{-1} \mathcal{L}_{v} \bar{g} & =\operatorname{det} L \cdot L g^{-1} \mathcal{L}_{v}\left(\frac{1}{\operatorname{det} L} g L^{-1}\right) \\
& =-\mathcal{L}_{v}(\log |\operatorname{det} L|) \cdot \operatorname{Id}+L g^{-1} \mathcal{L}_{v} g L^{-1}-\mathcal{L}_{v}(L) \cdot L^{-1} .
\end{aligned}
$$

Substituting (17) to the left-hand side and (16) to the right-hand side (into the middle term) of this relation, we get

$$
\begin{array}{r}
-\mathcal{L}_{v}(\log (|\operatorname{det}(L)|)) \cdot \operatorname{Id}+a \cdot L+(a \cdot \operatorname{tr}(L)+(n+1) b) \cdot \operatorname{Id}-\mathcal{L}_{v}(L) \cdot L^{-1} \\
=c L^{-1}+\left(c \cdot \operatorname{tr}\left(L^{-1}\right)+(n+1) d\right) \cdot \operatorname{Id} .
\end{array}
$$

After multiplication by $L$, this relation can be rewritten as $\mathcal{L}_{v}(L)=a \cdot L^{2}+\left(-\mathcal{L}_{v}(\log |\operatorname{det} L|)+a \operatorname{tr} L-c \operatorname{tr} L^{-1}+(n+1)(b-d)\right) \cdot L-c \cdot \operatorname{Id}$, which is equivalent to (15).

Lemma 3. Let $\chi=\chi_{1} \cdot \chi_{2}$ be an admissible factorisation of the characteristic polynomial of $L$ and let $\left(x^{1}, \ldots, x^{r}, y^{r+1}, \ldots, y^{n}\right)$ be the local coordinate system induced by this factorisation (see Theorems 1, 21). Then the projective vector field $v$ splits in this coordinate system in the sense that

$$
v=\sum_{i=1}^{r} v^{i}(x) \frac{\partial}{\partial x^{i}}+\sum_{j=r+1}^{n} v^{j}(y) \frac{\partial}{\partial y^{j}},
$$

i.e., the first $r$ entries of $v$ do not depend on the $y$-coordinates, and the last $n-r$ entries of $v$ do not depend on the $x$-coordinates. Moreover, the function $\beta$ from Lemma 2 is constant.

Proof. By Theorems 1, 2, in the coordinate system $(\bar{x}, \bar{y})$, the matrix of $L$ has the block-diagonal form (4). Then, the matrices of $L^{2}$ and of $\mathcal{L}_{v}(\log |\operatorname{det} L|) \cdot L$ are block-diagonal as well with the same dimensions of blocks implying that $\mathcal{L}_{v}(L)$ is also block-diagonal. Then, the first $r$ components of $v$ depend on $x$-coordinates only, and the last $(n-r)$-components of $v$ depend on $y$-coordinates only: $v=$ $\left(v_{1}(x), v_{2}(y)\right)$ where $v_{1} \in T B_{1}$ and $v_{2} \in T B_{2}$. Multiplying the equation (18) by $L^{-1}$ and rewriting it in the form

$$
\left(\mathcal{L}_{v} L\right) \cdot L^{-1}-\alpha \cdot L-\gamma \cdot L^{-1}=\beta \cdot \mathrm{Id},
$$

we see that the first block of the left-hand side is independent of $y$ and the second block of the left-hand side is independent of $x$, implying $\beta=$ const.

Thus, under the additional assumption that an admissible factorisation exists, the tensor $L$ and the vector field $v$ satisfy the following equation:

$$
\mathcal{L}_{v}(L)=\alpha \cdot L^{2}+\beta \cdot L+\gamma \cdot \operatorname{Id},
$$


where $\alpha, \gamma$ and $\beta$ are certain constants. Moreover, in the notation of Theorem 2 the $r \times r-$ resp. $(n-r) \times(n-r)-$ matrices $L_{1}$ and $L_{2}$ viewed as $(1,1)$-tensors satisfy the equations

$$
\begin{aligned}
& \mathcal{L}_{v_{1}}\left(L_{1}\right)=\alpha \cdot L_{1}^{2}+\beta \cdot L_{1}+\gamma \cdot \mathrm{Id}, \\
& \mathcal{L}_{v_{2}}\left(L_{2}\right)=\alpha \cdot L_{2}^{2}+\beta \cdot L_{2}+\gamma \cdot \mathrm{Id},
\end{aligned}
$$

where the components of the vector $v_{1}$ are the first $r$ entries of $v$, and the components of the vector $v_{2}$ are the last $n-r$ entries of $v$. In other words, equation (18) splits into two independent equations (19), (20).

We can go further: if $\chi$ is a product of several mutually prime monic polynomials $\chi_{1}, \ldots, \chi_{m}$, we can split the equation (20) into $m$ equations of the similar form by repeating the above construction. After finitely many steps, we land at an independent system of PDE of the form

$$
\mathcal{L}_{v_{i}}\left(L_{i}\right)=\alpha \cdot L_{i}^{2}+\beta \cdot L_{i}+\gamma \cdot \mathrm{Id}, \quad i=1, \ldots, m
$$

where each $L_{i}$ and $v_{i}$ depend on the corresponding coordinates only. Moreover, in a neighborhood of a regular point we may assume that $L_{i}$ has one real eigenvalue, or two complex-conjugate eigenvalues.

Thus, it is sufficient to solve equation (18) under the assumption that $L$ has one real eigenvalue, or two complex-conjugate eigenvalues.

In the Riemannian case, equation (18) was obtained by other methods and played an important role in the proof of the projective Lichnerowicz Conjecture [Ma5], and in the local description of projective vector fields [Fu, So, BMM, Ma7]. This equation is relatively simple and can be solved in the Riemannian case explicitly. The analysis of its solutions was, in fact, one of the most principal steps in the solution of the Lie Problem and of the Lichnerowicz Conjecture (under the additional assumption that the degree of mobility is at most two).

We expect similar applications in the pseudo-Riemannian case. If $L$ is semisimple and has real eigenvalues, then at least locally there is no essential difference from the Riemannian case. The possible difficulties might appear if $L$ has Jordan blocks or complex-conjugated eigenvalues. They already appear in dimension 2 (though in dimension 2 the difficulties have been overcome): two-dimensional metrics admitting projective vector fields in the case when $L$ is a Jordan block are given by much more complicated formulas than those which appear for $L$ semi-simple; see [Ma7, Theorem 1].

2.3. Motivation III. Topological geodesic rigidity problem. A local version of the splitting/gluing constructions is sufficient for local problems such as finding normal forms for a pair of geodesically equivalent metrics and the Lie Problem. For global problems (when the underlying manifold is assumed to be closed and/or complete) such as the Lichnerowicz Conjecture discussed above, a general version of the splitting/gluing constructions was useful in the Riemannian case, and is expected to be useful in the pseudo-Riemannian one. Another example of global problems is

Topological geodesic rigidity problem. On what manifolds, do the unparameterized geodesics of any metric $g$ determine this metric uniquely (up to multiplication by a constant)? 
In other words, we want to know whether or not a given manifold $M$ admits at least one pair $g \sim \bar{g}$ of nonproportional geodesically equivalent (pseudo)Riemannian metrics.

All results in this area are, in fact, very recent: the first obstructions that prevent a manifold from possessing such metrics were found in [MT1] (it was proved that geodesically equivalent metrics on a closed surface of genus $\geq 2$ are always proportional). Moreover, it was generally believed and explicitly stated in the survey Mi2 that it is hard to obtain such obstructions. The main research was concentrated in the following direction: find out what metrics are geodesically rigid, in the sense that they do not possess nontrivial geodesically equivalent "partners". One of the first results in this direction is $[\mathrm{Si2}$ : every metric geodesically equivalent to an irreducible symmetric metric of nonconstant curvature is proportional to it. The local geodesic rigidity problem was very popular in the 1960s to the 1980s; there are more than 100 papers devoted to it; see surveys [Mi2, Am2.

Later, one started to investigate the global geodesic rigidity problem (assuming that $M$ is closed or $g$ is complete). A typical result is as follows: if $g$ is a Riemannian Einstein irreducible metric of nonconstant curvature on a closed manifold, then it is geodesically rigid Mi1, Mi2 (though locally there exist Einstein Riemannian metrics of nonconstant curvature that are not geodesically rigid). The most standard (de facto, the only) way to prove such results was to use tensor calculus to canonically obtain a nonconstant function $f$ such that $\Delta_{g} f=$ const $\cdot f$ with const $\geq 0$, which of course cannot exist on a closed Riemannian manifold.

Here is one of the examples that shows how the splitting/gluing construction works in a global setting without any specific assumptions about the metric (we simply combine Theorem 3 with the results by $\mathrm{Wu}[\mathrm{Wu}$ and de Rham [DR]).

Proposition 2. Let $M$ admit a pair of geodesically equivalent (pseudo)-Riemannian metrics $g$ and $\bar{g}$, one of which is complete. Assume that the characteristic polynomial $\chi$ of $L=L(g, \bar{g})$ possesses an admissible global factorisation $\chi=\chi_{1} \cdot \chi_{2}$ on $M$. Then $M$ admits a (pseudo)-Riemannian metric $h$ with a reducible holonomy group, and its universal cover $\left(\widetilde{M}, \pi^{*} h\right)$ (where $\pi: \widetilde{M} \rightarrow M$ is the natural projection) is the direct product of two (pseudo)-Riemannian manifolds $\left(M_{1}, h_{1}\right)$ and $\left(M_{2}, h_{2}\right)$ such that the fundamental group $\pi_{1}(M)$ acts on $M_{1} \times M_{2}$ by fiberwise isometries.

Obviously, the above property is a very strong topological restriction on $M$. For example, in dimension 2 , there are only five connected manifolds of this kind: $\mathbb{R}^{2}$, torus, Klein bottle, Möbius strip and cylinder $\mathbb{R}^{1} \times S^{1}$.

The additional assumption in Proposition 2 about the existence of a global admissible factorisation is, of course, very essential. There are many examples of geodesically equivalent metrics where this condition fails. It appears, however, that in the Riemannian case the nonexistence of such a factorisation implies that the fundamental group of $M$ is finite (assuming that $M$ is closed and admits two nonproportional geodesically equivalent metrics) Ma1.

Combining these two observations, it was possible to describe all geodesically rigid 3-manifolds [Ma2, and to prove that topologically-hyperbolic manifolds are geodesically rigid, in the sense that two geodesically equivalent metrics on such manifolds are proportional Ma1.

At the present point, it is not clear whether the second observation could be generalised to the pseudo-Riemannian case, i.e., whether the nonexistence of an admissible factorisation implies that the fundamental group is finite. However, the 
known examples allow us to suggest that compared to the classical Riemannian situation, the topological obstructions to the existence of geodesically equivalent metrics are perhaps even stronger in the pseudo-Riemannian case. We conclude this section by the following

Conjecture. Let $g \sim \bar{g}$ be geodesically equivalent nonproportional metrics on a closed 3-dimensional manifold $M^{3}$. If at least one of the metrics is complete, and at least one of the metrics has signature $(-,+,+)$, then $M$ is a Seifert manifold with zero Euler class.

\section{Proofs}

3.1. Vanishing of Nijenhuis torsion and proof of Theorems 1, 2, Throughout the paper we shall use the following analytic condition for two metrics $g$ and $\bar{g}$ to be geodesically equivalent.

Proposition 3. Let $g, \bar{g}$ be Riemannian or pseudo-Riemannian metrics on the same manifold $M^{n}$. Let $L=L(g, \bar{g})$ be given by (11).

Then, $g$ and $\bar{g}$ are geodesically equivalent if and only if

$$
\nabla_{u} L=\frac{1}{2}\left(u \otimes l+(u \otimes l)^{*}\right) \quad \text { for any tangent vector } u,
$$

or, in coordinates,

$$
\nabla_{r} L_{q}^{p}=\frac{1}{2}\left(\delta_{r}^{p} l_{q}+g^{p s} l_{s} g_{r q}\right)
$$

where $\nabla$ is the Levi-Civita connection associated with $g, l=d \operatorname{tr} L$ and $C^{*}$ denotes the operator $g$-adjoint to $C$, i.e., $g(C u, v)=g\left(u, C^{*} v\right)$.

The above proposition and equation (21) are due to Sinjukov [Si1]; the selfcontained proof can also be found in [BM, EM].

Since $\bar{g}$ can be uniquely reconstructed from $g$ and $L$ as $\bar{g}=\frac{1}{\operatorname{det} L} g L^{-1}$, we may replace the pair of metrics $(g, \bar{g})$ by the pair $(g, L)$. For convenience, we shall say that a metric $g$ and a $g$-selfadjoint nondegenerate $(1,1)$-tensor $L$ are compatible if they satisfy (21), so that the compatibility of $g$ and $L$ is just rephrasing the fact that $g$ and $\bar{g}$ are geodesically equivalent.

First of all we recall that the compatibility of $g$ and $L$ implies that the Nijenhuis torsion of $L$ vanishes identically; see [BM]. To make our paper self-contained, we recall some basic facts about the Nijenhuis torsion and prove that $N_{L} \equiv 0$ for any $(1,1)$-tensor $L$ satisfying (21).

The Nijenhuis torsion of $L$ is the (1,2)-tensor field defined by

$$
N_{L}(u, v)=L^{2}[u, v]-L[L u, v]-L[u, L v]+[L u, L v],
$$

where $u$ and $v$ are vector fields on $M$ [Haa]. This definition immediately implies

Lemma 4. The condition $N_{L}=0$ admits the following equivalent forms:

(1) $\mathcal{L}_{L u} L-L \mathcal{L}_{u} L=0$ for any vector field $u$, where $\mathcal{L}_{u}$ is the Lie derivative along $u$;

(2) $\left(\nabla_{L u} L-L \nabla_{u} L\right) v$ is symmetric with respect to $u$ and $v$ for any vector fields $u$ and $v$, where $\nabla$ is the Levi-Civita connection of an arbitrary metric $g$ (or, more generally, any symmetric connection).

Lemma 5 ([BM]). If $L$ satisfies (21), then $N_{L}=0$. 
Proof. By Lemma 4 , we just need to verify that $\left(\nabla_{L u} L-L \nabla_{u} L\right) v$ is symmetric with respect to $u$ and $v$. We simply use the compatibility condition (21):

$$
\begin{aligned}
& \left(\nabla_{L u} L-L \nabla_{u} L\right) v=\frac{1}{2}\left((L u \otimes l) v+(L u \otimes l)^{*} v-L(u \otimes l) v-L(u \otimes l)^{*} v\right) \\
& =\frac{1}{2}\left(l(v) \cdot L u+g(L u, v) \cdot g^{-1}(l)-l(v) \cdot L u-g(u, v) \cdot L\left(g^{-1}(l)\right)\right) \\
& =\frac{1}{2}\left(g(L u, v) \cdot g^{-1}(l)-g(u, v) \cdot L\left(g^{-1}(l)\right)\right) .
\end{aligned}
$$

Here $g^{-1}$ is viewed as the identification map between $T_{x}^{*} M$ and $T_{x} M$; in particular, $g^{-1}(l)=\operatorname{grad} \operatorname{tr} L$. The symmetry of the last expression with respect to $u$ and $v$ is now evident.

The next two statements are well known in folklore; however, we could not find any reference with a short proof.

Lemma 6. If $N_{L}=0$, then $N_{p(L)}=0$ for any polynomial $p: \mathbb{R} \rightarrow \mathbb{R}$ (with constant real coefficients) and, therefore, $N_{f(L)}=0$ for any function $f: K \rightarrow \mathbb{C}$ satisfying the assumptions (ii) -(可) of Section 1.3 .

Proof. The latter statement about $f(L)$ follows immediately from the definition of $f(L)$. The proof for a polynomial $p(L)$ is as follows. We use the condition $N_{L}=0$ in the form $\mathcal{L}_{L u} L=L \mathcal{L}_{u} L$ (see Lemma 4). This identity implies that

$$
\mathcal{L}_{L^{n} u} L=\mathcal{L}_{L\left(L^{n-1} u\right)} L=L \mathcal{L}_{L^{n-1} u} L=L \mathcal{L}_{L\left(L^{n-2} u\right)} L=L^{2} \mathcal{L}_{L^{n-2} u} L=\cdots=L^{n} \mathcal{L}_{u} L,
$$

and, therefore, by linearity,

$$
\mathcal{L}_{p(L) u} L=p(L) \mathcal{L}_{u} L
$$

Thus, we have

$$
\left(\mathcal{L}_{p(L) u}-p(L) \mathcal{L}_{u}\right) L=0
$$

Now consider the expression $\mathcal{D}=\mathcal{L}_{p(L) u}-p(L) \mathcal{L}_{u}$ as a "first-order differential operator" which satisfies the obvious property $\mathcal{D}\left(L^{n}\right)=\mathcal{D}\left(L^{n-1}\right) L+L^{n-1} \mathcal{D}(L)$. Hence, the identity $\mathcal{D}(L)=0$ immediately implies $\mathcal{D}(p(L))=0$, i.e.,

$$
\left(\mathcal{L}_{p(L) u}-p(L) \mathcal{L}_{u}\right) p(L)=0,
$$

which is exactly the desired condition $N_{p(L)}=0$.

Lemma 7. Let $N_{L}=0$ and $\chi=\chi_{1} \cdot \chi_{2}$ be an admissible factorisation of the characteristic polynomial of $L$. Then there exists a local coordinate system $\left(x^{1}, \ldots, x^{r}, y^{r+1}\right.$, $\left.\ldots, y^{n}\right)$ such that $\partial_{x^{1}}, \ldots, \partial_{x^{r}}$ and $\partial_{y^{r+1}}, \ldots, \partial_{y^{n}}$ generate the kernels $D_{1}$ and $D_{2}$ of the operators $\chi_{1}(L)$ and $\chi_{2}(L)$, respectively, and

$$
L(x, y)=\left(\begin{array}{cc}
L_{1}(x) & 0 \\
0 & L_{2}(y)
\end{array}\right) .
$$

In particular, the distributions $D_{1}$ and $D_{2}$ are integrable.

Proof. At each tangent space $T_{x} M$ we have the natural decomposition $T_{x} M=$ $D_{1} \oplus D_{2}$, where $D_{i}=\operatorname{ker} \chi_{i}(L)$. This decomposition defines two natural projectors $P_{1}$ and $P_{2}$ onto the subspaces $D_{1}$ and $D_{2}$, respectively. A simple but important observation is that (locally) these projectors can be viewed as functions $P_{1}=f_{1}(L)$ and $P_{2}=f_{2}(L)$ satisfying the assumptions (ii) $-(\mathbb{\nabla})$ from Section 1.3 (see Example (4). 
Thus, by Lemma 6. $N_{P_{i}}=0$, and we will use this fact to prove the integrability of $D_{1}$ and $D_{2}$. In terms of the projectors $P_{1}$ and $P_{2}$, these distributions can obviously be interpreted as $D_{1}=\operatorname{ker} P_{2}$ and $D_{2}=\operatorname{ker} P_{1}$. Let $u, v \in D_{1}=\operatorname{ker} P_{2}$. Then the condition $N_{P_{2}}=0$ gives

$$
P_{2}^{2}[u, v]-P_{2}\left[P_{2} u, v\right]-P_{2}\left[u, P_{2} v\right]+\left[P_{2} u, P_{2} v\right]=P_{2}^{2}[u, v]=0,
$$

that is, $[u, v] \in \operatorname{ker} P_{2}^{2}=\operatorname{ker} P_{2}=D_{1}$, which is equivalent to the integrability of $D_{1}$ by the Frobenius Theorem. The same is obviously true for $D_{2}$ by the same reason.

The integrability of these two distributions is equivalent to the existence of a coordinate system $(x, y)$ such that $\partial_{x^{1}}, \ldots, \partial_{x^{r}}$ and $\partial_{y^{r+1}}, \ldots, \partial_{y^{n}}$ generate the kernels of the operators $\chi_{1}(L)$ and $\chi_{2}(L)$. In particular, the operator $L$ in this coordinate system has a block-diagonal form:

$$
L(x, y)=\left(\begin{array}{cc}
L_{1}(x, y) & 0 \\
0 & L_{2}(x, y)
\end{array}\right)
$$

Without loss of generality we may assume that $\operatorname{det} L \neq 0$; otherwise we can (locally) replace $L$ by $L+c \cdot \operatorname{Id}$, where $c$ is an appropriate constant.

Notice that the operator

$$
L P_{1}=\left(\begin{array}{cc}
L_{1}(x, y) & 0 \\
0 & 0
\end{array}\right),
$$

being a function of $L$, has zero Nijenhuis torsion. Thus, for $u=\partial_{y_{\alpha}} \in D_{2}=\operatorname{ker} P_{1}$ we have

$$
\begin{aligned}
\left(\begin{array}{ll}
0 & 0 \\
0 & 0
\end{array}\right) & =\mathcal{L}_{L P_{1} u}\left(L P_{1}\right)-L P_{1} \mathcal{L}_{u}\left(L P_{1}\right)=L P_{1} \mathcal{L}_{u}\left(L P_{1}\right) \\
& =\left(\begin{array}{cc}
L_{1} & 0 \\
0 & 0
\end{array}\right)\left(\begin{array}{cc}
\partial_{y_{\alpha}} L_{1} & 0 \\
0 & 0
\end{array}\right)=\left(\begin{array}{cc}
L_{1} \partial_{y_{\alpha}} L_{1} & 0 \\
0 & 0
\end{array}\right) .
\end{aligned}
$$

Since $L_{1}$ is nondegenerate, we conclude that $\partial_{y_{\alpha}} L_{1}=0$, i.e., $L_{1}=L_{1}(x)$. Similarly, $L_{2}=L_{2}(y)$, as needed.

Proof of Theorems 1, 2, The statements of these theorems are straightforward from Lemmas 5, 7.

3.2. Proof of the generalised Topalov-Sinjukov Theorem [5, Consider two geodesically equivalent metrics $g$ and $\bar{g}$ and assume that $L$ given by (1) and a function $f: K \rightarrow \mathbb{C}$ satisfy the assumptions (ii)-(미) of Section 1.3. Our goal is to prove Theorem [ 5 i.e., to show that the metric $g_{f}:=g f(L)$ is compatible with $L$. To simplify our notation below, we shall denote $g_{f}$ by $\widetilde{g}$.

We need to verify that the main equation (21) for $g$ and $L$ implies the similar relation for $\widetilde{g}=g f(L)$ and $L$ :

$$
\widetilde{\nabla}_{u} L=\frac{1}{2}\left(u \otimes l+(u \otimes l)^{\widetilde{*}}\right),
$$

where $C^{\widetilde{*}}$ denotes the operator $\widetilde{g}$-adjoint of $C$, and $\widetilde{\nabla}$ is the covariant differentiation with respect to the Levi-Civita connection related to $\widetilde{g}$.

First of all, we rewrite the condition that we should verify in a slightly different way by subtracting (21) from (22):

$$
\left(\widetilde{\nabla}_{u}-\nabla_{u}\right) L=\frac{1}{2}\left((u \otimes l)^{*}-(u \otimes l)^{\widetilde{*}}\right) .
$$


Now notice that the difference of two covariant derivatives on the left-hand side is not a differential operator, but a tensor expression of the form

$$
\left(\widetilde{\nabla}_{u}-\nabla_{u}\right) L=T_{u} L-L T_{u}
$$

where $\left(T_{u}\right)_{j}^{i}=T_{j k}^{i} u^{k}$ and $T_{j k}^{i}=\Gamma_{j k}^{i}-\widetilde{\Gamma}_{j k}^{i}$ is a $(1,2)$-tensor that represents the difference of the two connections related to $g$ and $\widetilde{g}$.

Using the obvious fact that $C^{\widetilde{*}}=f(L)^{-1} C^{*} f(L)$ for any operator $C$, we can rewrite (23) as:

$$
T_{u} L-L T_{u}=\frac{1}{2}\left((u \otimes l)^{*}-f(L)^{-1}(u \otimes l)^{*} f(L)\right) .
$$

Finally multiplying both sides by $f(L)$ from the left, we see that the statement of the Topalov-Sinjukov Theorem is equivalent to the following algebraic relation:

$$
\left[f(L) T_{u}, L\right]=\frac{1}{2}\left[f(L),(u \otimes l)^{*}\right],
$$

where $[\cdot, \cdot]$ denotes the standard commutator of linear operators.

To verify it, we compute the tensor $T_{u}$ explicitly, using the following equation:

$$
T_{j k}^{s} \widetilde{g}_{s i}+T_{k i}^{s} \widetilde{g}_{s j}=\left(\widetilde{\nabla}_{k}-\nabla_{k}\right) \widetilde{g}_{i j}=-\nabla_{k} \widetilde{g}_{i j} \text {. }
$$

This is a system of linear equations w.r.t. $T_{j k}^{s} \widetilde{g}_{s i}$ with the well-known unique solution:

or, in invariant terms,

$$
T_{j k}^{s} \widetilde{g}_{s i}=\frac{1}{2}\left(\nabla_{j} \widetilde{g}_{s k}+\nabla_{k} \widetilde{g}_{s j}-\nabla_{s} \widetilde{g}_{j k}\right),
$$

$$
\widetilde{g}\left(T_{u} v, w\right)=\frac{1}{2}\left(\left(\nabla_{u} \widetilde{g}\right)(v, w)+\left(\nabla_{v} \widetilde{g}\right)(u, w)-\left(\nabla_{w} \widetilde{g}\right)(u, v)\right) .
$$

In our particular case, we have:

$$
\begin{aligned}
\nabla_{u} \widetilde{g}=g \nabla_{u} f(L) & =g f_{t=0}^{\prime}\left(L+t \nabla_{u} L\right) \\
& =f_{t=0}^{\prime}\left(L+t \frac{1}{2}\left(u \otimes l+(u \otimes l)^{*}\right)\right) \\
& =\frac{1}{2} g f_{t=0}^{\prime}(L+t(u \otimes l))+\frac{1}{2} g f_{t=0}^{\prime}\left(L+t(u \otimes l)^{*}\right)=\frac{1}{2} g\left(A_{u}+A_{u}^{*}\right),
\end{aligned}
$$

where $A_{u}=f_{t=0}^{\prime}(L+t(u \otimes l))$ and $A_{u}^{*}=f_{t=0}^{\prime}\left(L+t(u \otimes l)^{*}\right)$. The operators $A_{u}$ and $A_{u}^{*}$ are $g$-adjoint to each other and satisfy the following important property.

Lemma 8. In the notation above, $A_{u}^{*} v=A_{v}^{*} u$.

Proof. If $f(L)=L$, then $A_{u}^{*}=(u \otimes l)^{*}=g^{-1}(l) \otimes g(u)$ and we have $A_{u}^{*} v=$ $g(u, v) g^{-1}(l)=A_{v}^{*} u$ (here we consider $g$ and $g^{-1}$ as identification operators between the tangent and cotangent spaces).

If $f(L)=L^{2}$, then $A_{u}^{*}=L(u \otimes l)^{*}+(u \otimes l)^{*} L$ and $A_{u}^{*} v=g(u, v) \cdot L g^{-1}(l)+$ $g(u, L v) \cdot g^{-1}(l)=A_{v}^{*} u$ (we use, of course, the fact that $L$ is $g$-selfadjoint).

More generally, for $f(L)=L^{n}$, we have

$$
A_{u}^{*} v=\sum_{m=0}^{n-1} g\left(L^{n-m} u, v\right) \cdot L^{m-1} g^{-1}(l)=A_{v}^{*} u .
$$

Thus, the statement holds for any polynomial $f(L)$ and, therefore, for any smooth function $f$ satisfying (ii)-(可) in Section 1.3 . 
Using these properties, we get a surprisingly simple result by substituting (26) into (25):

$$
\begin{aligned}
\widetilde{g}\left(T_{u} v, w\right) & =\frac{1}{4}\left(g\left(\left(A_{u}+A_{u}^{*}\right) v, w\right)+g\left(\left(A_{v}+A_{v}^{*}\right) u, w\right),\right. \\
\left.-g\left(\left(A_{w}+A_{w}^{*}\right) u, v\right)\right) & =\frac{1}{2} g\left(A_{u}^{*} v, w\right) .
\end{aligned}
$$

In other words, $f(L) T_{u}=A_{u}^{*}=\frac{1}{2} f_{t=0}^{\prime}\left(L+t(u \otimes l)_{g}^{*}\right)$.

We are now ready to complete the proof. We use the following simple matrix relation, which obviously holds for any $L, B$ and $f$ :

$$
0=[f(L+t B), L+t B]_{t=0}^{\prime}=\left[f_{t=0}^{\prime}(L+t B), L\right]+[f(L), B],
$$

that is,

$$
\left[f_{t=0}^{\prime}(L+t B), L\right]=[B, f(L)] .
$$

In our case $B=(u \otimes l)_{g}^{*}$ and $f_{t=0}^{\prime}(L+t B)=2 f(L) T_{u}$ which gives exactly (24), as required.

3.3. Proof of Theorem 3. Theorems 1, 2 reduce our consideration to the blockdiagonal case in the sense that the metric $g$ and operator $L$ both have block-diagonal form in local coordinates $(x, y)=\left(x^{1}, \ldots, x^{r}, y^{r+1}, \ldots, y^{n}\right)$; more precisely,

$$
g=\left(\begin{array}{cc}
g_{1}(x, y) & 0 \\
0 & g_{2}(x, y)
\end{array}\right), \quad L=\left(\begin{array}{cc}
L_{1}(x) & 0 \\
0 & L_{2}(y)
\end{array}\right) .
$$

Notice first that in such a situation, the main equation (21) has a rather special form. Namely, it can naturally be divided into three parts, each of which has its own meaning and can be treated separately (up to some extent).

Index notation convention. For convenience, throughout the rest of the paper we denote the indices for $x^{1}, \ldots, x^{r}$ by Latin letters $i, j, k, l, m=1, \ldots, r$, and those for $y^{r+1}, \ldots, y^{n}$ by Greek letters $\alpha, \beta, \gamma, \delta=r+1, \ldots, n$. The indices $p, q, r, s, t$ will serve for both cases, i.e., $p, q, r, s, t=1, \ldots, n$.

Let $u \in D_{1}$. Then the main equation (21) can be written in a block form as

$$
\left(\begin{array}{cc}
\nabla_{u} L_{j}^{i} & \nabla_{u} L_{\alpha}^{i} \\
\nabla_{u} L_{j}^{\beta} & \nabla_{u} L_{\alpha}^{\beta}
\end{array}\right)=\left(\begin{array}{cc}
\frac{1}{2}\left(u^{i} \frac{\partial \operatorname{tr} L}{\partial x^{j}}+g^{i l} \frac{\partial \operatorname{tr} L}{\partial x^{l}} u^{m} g_{m j}\right) & \frac{1}{2} u^{i} \frac{\partial \operatorname{tr} L}{\partial y^{\alpha}} \\
\frac{1}{2} g^{\beta \gamma} \frac{\partial \operatorname{tr} L}{\partial y^{\gamma}} u^{m} g_{m j} & 0
\end{array}\right) .
$$

We rewrite it for each block separately, taking into account the block-diagonal form of $g$ and $L$ and the fact that $\frac{\partial \operatorname{tr} L}{\partial x^{l}}=\frac{\partial \operatorname{tr} L_{1}}{\partial x^{l}}$ and $\frac{\partial \operatorname{tr} L}{\partial y^{\alpha}}=\frac{\partial \operatorname{tr} L_{2}}{\partial y^{\alpha}}$ :

$$
\begin{aligned}
& u^{k} \frac{\partial\left(L_{1}\right)_{j}^{i}}{\partial x^{k}}+u^{k} \Gamma_{k m}^{i}\left(L_{1}\right)_{j}^{m}-u^{k} \Gamma_{k j}^{m}\left(L_{1}\right)_{m}^{i} \\
& =\frac{1}{2}\left(u^{i} \frac{\partial \operatorname{tr} L_{1}}{\partial x^{j}}+\left(g_{1}\right)^{i l} \frac{\partial \operatorname{tr} L_{1}}{\partial x^{l}} u^{m}\left(g_{1}\right)_{m j}\right), \\
& u^{k} \Gamma_{k \beta}^{j}\left(L_{2}\right)_{\alpha}^{\beta}-u^{k} \Gamma_{k \alpha}^{m}\left(L_{1}\right)_{m}^{j}=\frac{1}{2} u^{j} \frac{\partial \operatorname{tr} L_{2}}{\partial y^{\alpha}} \\
& u^{k} \Gamma_{\gamma k}^{\alpha}\left(L_{2}\right)_{\beta}^{\gamma}-\left(L_{2}\right)_{\gamma}^{\alpha} u^{k} \Gamma_{\beta k}^{\gamma}=0 .
\end{aligned}
$$

We omit the equation for $\nabla_{u} L_{j}^{\beta}$ because it is obtained from (29) by using the fact that the left-hand side and right-hand side on (21) are both $g$-selfadjoint.

First of all, we notice that the Christoffel symbols $\Gamma_{j k}^{i}(i, j, k=1, \ldots, r<n)$ of the Levi-Civita connection associated with $g$ coincide with those for the metric $g_{1}$ defined on the leaves of the integrable distribution $D_{1}$. Thus, the first equation 
(28) simply means that $g_{1}(x, y)$ and $L_{1}(x)$ are compatible on each leaf of $D_{1}$, i.e., for every fixed $y$.

To simplify (29) and (30), we notice that

$$
\Gamma_{\alpha k}^{i}=\frac{1}{2} g^{i m} \frac{\partial g_{m k}}{\partial y^{\alpha}} \quad \text { and } \quad \Gamma_{\alpha k}^{\beta}=\frac{1}{2} g^{\beta \gamma} \frac{\partial g_{\gamma \alpha}}{\partial x^{k}} .
$$

Substituting these expressions into (29) and (30) and taking into account that $u \in D_{1}$ is an arbitrary vector, we obtain

$$
g^{j m} \frac{\partial g_{m k}}{\partial y^{\beta}}\left(L_{2}\right)_{\alpha}^{\beta}-\left(L_{1}\right)_{m}^{j} g^{m l} \frac{\partial g_{l k}}{\partial y^{\alpha}}=\delta_{k}^{j} \frac{\partial \operatorname{tr} L_{2}}{\partial y^{\alpha}}
$$

and

$$
g^{\alpha \beta} \frac{\partial g_{\beta \gamma}}{\partial x^{k}}\left(L_{2}\right)_{\beta}^{\gamma}-\left(L_{2}\right)_{\gamma}^{\alpha} g^{\gamma \beta} \frac{\partial g_{\beta \alpha}}{\partial x^{k}}=0 .
$$

Summarizing this discussion and rewriting (31) and (32) in a shorter "matrix" form, we obtain

Proposition 4. For $g$ and $L$ of block-diagonal form (27), the compatibility equation (21) for $u \in D_{1}$ is equivalent to the following three conditions:

Condition 1. $g_{1}(x, y)$ and $L_{1}(x)$ are compatible on each leaf of $B_{1}$ (here $x$ are coordinates on a leaf, and $y$ is a parameter which determines the leaf);

\section{Condition 2.}

$$
\left(g_{1}^{-1} d_{y} g_{1}\right) L_{2}-L_{1}\left(g_{1}^{-1} d_{y} g_{1}\right)=\operatorname{Id}_{D_{1}} \otimes d_{y} \operatorname{tr} L_{2}
$$

\section{Condition 3.}

$$
\left(g_{2}^{-1} d_{x} g_{2}\right) L_{2}-L_{2}\left(g_{2}^{-1} d_{x} g_{2}\right)=0 .
$$

As we shall now see, Theorems 3 and 4 are algebraic corollaries of these equations, the generalized Topalov-Sinjukov Theorem 5 and the vanishing of $N_{L}$.

The first part of Theorem 3 states that the metric

$$
h=\left(\begin{array}{cc}
h_{1} & 0 \\
0 & h_{2}
\end{array}\right)=\left(\begin{array}{cc}
g_{1} \chi_{2}\left(L_{1}\right)^{-1} & 0 \\
0 & g_{2} \chi_{1}\left(L_{2}\right)^{-1}
\end{array}\right)
$$

is of local product structure. In other words, we need to check that

$$
\frac{\partial\left(h_{1}\right)_{l m}}{\partial y^{\alpha}}=0 \quad \text { and } \quad \frac{\partial\left(h_{2}\right)_{\alpha \beta}}{\partial x^{m}}=0 .
$$

We shall verify the first condition only; the proof for the second is similar. We start with a straightforward computation.

Lemma 9. Let $h_{1}=g_{1} \chi_{2}\left(L_{1}\right)^{-1}$. Then

$$
\frac{\partial\left(h_{1}\right)_{l m}}{\partial y_{\alpha}}=g_{l i}\left(g^{i j} \frac{\partial g_{j k}}{\partial y^{\alpha}}-\left(\chi_{2}\left(L_{1}\right)^{-1}\right)_{j}^{i} \frac{\partial \chi_{2}\left(L_{1}\right)_{k}^{j}}{\partial y^{\alpha}}\right)\left(\chi_{2}\left(L_{1}\right)^{-1}\right)_{m}^{k}
$$

or, in coordinate-free form,

$$
d_{y} h_{1}=g_{1}\left(g_{1}^{-1} d_{y} g_{1}-\chi_{2}\left(L_{1}\right)^{-1} d_{y} \chi_{2}\left(L_{1}\right)\right) \chi_{2}\left(L_{1}\right)^{-1} .
$$


Proof. Thinking of $h_{1}=g_{1} \chi_{2}\left(L_{1}\right)^{-1}$ as a product of two $r \times r$ matrices, we have

$$
\begin{aligned}
\frac{\partial}{\partial y_{\alpha}} h_{1} & =\frac{\partial}{\partial y^{\alpha}}\left(g_{1} \chi_{2}\left(L_{1}\right)^{-1}\right)=\frac{\partial g_{1}}{\partial y^{\alpha}} \chi_{2}\left(L_{1}\right)^{-1}+g_{1} \frac{\partial \chi_{2}\left(L_{1}\right)^{-1}}{\partial y^{\alpha}} \\
& =\frac{\partial g_{1}}{\partial y^{\alpha}} \chi_{2}\left(L_{1}\right)^{-1}-g_{1} \chi_{2}\left(L_{1}\right)^{-1} \frac{\partial \chi_{2}\left(L_{1}\right)}{\partial y^{\alpha}} \chi_{2}\left(L_{1}\right)^{-1} \\
& =g_{1}\left(g_{1}^{-1} \frac{\partial g_{1}}{\partial y^{\alpha}}-\chi_{2}\left(L_{1}\right)^{-1} \frac{\partial \chi_{2}\left(L_{1}\right)}{\partial y^{\alpha}}\right) \chi_{2}\left(L_{1}\right)^{-1}
\end{aligned}
$$

as needed.

Thus, we need to prove that the expression in brackets on the left-hand side of (35) (or (36)) is actually zero. To do so, we need some properties of the differential of the characteristic polynomial of $L$ in the case when $N_{L}$ vanishes.

Lemma 10. Let $L$ be a tensor with zero Nijenhuis torsion, $l=d \operatorname{tr} L$ be the differential of $\operatorname{tr} L$ viewed as a covector and $\chi(t)=\operatorname{det}(t \cdot \operatorname{Id}-L)$ be the characteristic polynomial of $L$ viewed as a smooth function on $M$ with $t$ as a formal parameter. Then the differential of $\chi(t)$ satisfies the following relation:

$$
d \chi(t) L-t \cdot d \chi(t)=\chi(t) \cdot l .
$$

Remark 8. In (37), the right-hand and left-hand sides are both covectors, i.e. elements of the cotangent space. The expression $d \chi(t) L$ means that we apply the operator $L$ to the covector $d \chi$ using right multiplication. In coordinates, this means that

$$
(d \chi(t) L)_{s}=\frac{\partial \chi(t)}{\partial x^{p}} L_{s}^{p}
$$

The multiplication denoted by $\cdot$ simply means multiplying a covector by a scalar function.

Proof of Lemma 10. The differential of the polynomial

$$
\chi(t)=\operatorname{det}(t \cdot \operatorname{Id}-L)=a_{0}+a_{1} t+a_{2} t^{2}+\ldots+t^{n}
$$

is defined to be

$$
d \chi(t)=d a_{0}+t \cdot d a_{1}+t^{2} \cdot d a_{2}+\ldots+t^{n-1} \cdot d a_{n-1} .
$$

Instead of differentiating each coefficient separately, we are going to differentiate the whole polynomial (thinking of $t$ as a certain constant). For the operators $L$ with $N_{L}=0$, the following property is well known (see, for example, [BM, Lemma1]):

$$
(d \log |\operatorname{det} L|) L=d \operatorname{tr} L=l .
$$

Notice that $N_{t \cdot \operatorname{Id}-L}=0$ and apply the above identity to $\chi(t)=\operatorname{det}(t \cdot \operatorname{Id}-L)$ :

$$
(d \log |\chi(t)|)(t \cdot \operatorname{Id}-L)=d \operatorname{tr}(t \cdot \operatorname{Id}-L)=-d \operatorname{tr} L=-l .
$$

Thus $\frac{1}{\chi(t)} d \chi(t)(L-t \cdot \mathrm{Id})=l$, which is equivalent to (37).

In fact, we need to compute the differential of the characteristic polynomial in the case when $L=L_{2}$ and $t$ is replaced by $L_{1}$. More precisely we shall need the expression:

$$
\left(d_{y} \chi_{2}\left(L_{1}\right)\right)_{j, \alpha}^{i}=\frac{\partial}{\partial y^{\alpha}}\left(\chi_{2}\left(L_{1}\right)\right)_{j}^{i} .
$$


This object can be viewed naturally as an element of the space $\operatorname{Hom}\left(D_{1}, D_{1}\right) \otimes$ $D_{2}^{*}$. Since (37) is purely algebraic (in the sense that the nature of $t$ is not important) and $t=L_{1}$ does not depend on $y$, we can reformulate (37) for our special case as follows:

$$
d_{y} \chi_{2}\left(L_{1}\right) L_{2}-L_{1} d_{y} \chi_{2}\left(L_{1}\right)=\chi_{2}\left(L_{1}\right) \otimes l_{2}
$$

or, in coordinates,

$$
\left(d_{y} \chi_{2}\left(L_{1}\right)\right)_{j, \beta}^{i}\left(L_{2}\right)_{\alpha}^{\beta}-\left(L_{1}\right)_{k}^{i}\left(d_{y} \chi_{2}\left(L_{1}\right)\right)_{j, \alpha}^{k}=\left(\chi_{2}\left(L_{1}\right)\right)_{j}^{i}\left(l_{2}\right)_{\alpha},
$$

where $l_{2}=d \operatorname{tr} L_{2}$.

Multiplying both sides of (38) by $\chi_{2}\left(L_{1}\right)^{-1}$, and using that $L_{1}$ commutes with $\chi_{2}\left(L_{1}\right)$, we obtain

Lemma 11. If $N_{L}=0$, then the expression $\chi_{2}\left(L_{1}\right)^{-1} d_{y} \chi_{2}\left(L_{1}\right)$ satisfies the following relation:

$$
\left(\chi_{2}\left(L_{1}\right)^{-1} d_{y} \chi_{2}\left(L_{1}\right)\right) L_{2}-L_{1}\left(\chi_{2}\left(L_{1}\right)^{-1} d_{y} \chi_{2}\left(L_{1}\right)\right)=\operatorname{Id}_{D_{1}} \otimes l_{2}
$$

or, in coordinates,

$$
\left(\chi_{2}\left(L_{1}\right)^{-1}\right)_{m}^{j}\left(d_{y} \chi_{2}\left(L_{1}\right)\right)_{k, \beta}^{m}\left(L_{2}\right)_{\alpha}^{\beta}-\left(L_{1}\right)_{m}^{j}\left(\chi_{2}\left(L_{1}\right)^{-1}\right)_{l}^{m}\left(d_{y} \chi_{2}\left(L_{1}\right)\right)_{k, \alpha}^{l}=\delta_{k}^{j}\left(l_{2}\right)_{\alpha} .
$$

We are now ready to complete the proof of Theorem 3. Let $L$ and $g$ be compatible. Then Condition 2 from Proposition 4 holds. Comparing this condition (33) with the relation (39), we see that $\chi_{2}\left(L_{1}\right)^{-1} d_{y} \chi_{2}\left(L_{1}\right)$ and $g_{1}^{-1} d_{y} g_{1}$ satisfy the same relation. If we fix index $k$ in (33) and (39), we shall see that these relations can be treated as a linear matrix equation of the form

$$
X L_{2}-L_{1} X=C
$$

where $X$ is an (unknown) matrix of dimension $r \times(n-r)$, and $L_{1}, L_{2}, C$ are given matrices of dimensions $r \times r,(n-r) \times(n-r)$ and $r \times(n-r)$, respectively.

It is a simple fact in Linear Algebra that if $L_{1}$ and $L_{2}$ have no common eigenvalues, then the solution to this equation is unique for any $C$. Hence we conclude that

$$
\chi_{2}\left(L_{1}\right)^{-1} d_{y} \chi_{2}\left(L_{1}\right)=g_{1}^{-1} d_{y} g_{1},
$$

which immediately implies $\frac{\partial\left(h_{1}\right)_{i j}}{\partial y^{\alpha}}=0$ (see Lemma 9).

Analogously (i.e., just by interchanging the distributions $D_{1}$ and $D_{2}$ ), we can check that $\frac{\partial\left(h_{2}\right)_{\alpha \beta}}{\partial x^{i}}=0$. This means that in the metric

$$
h=\left(\begin{array}{cc}
h_{1} & 0 \\
0 & h_{2}
\end{array}\right)
$$

the block $h_{1}$ depends on the $x$-coordinates only, and the block $h_{2}$ depends on the $y$-coordinates only, i.e., $\left(h, B_{1}, B_{2}\right)$ is a local product structure. Thus, the first statement of Theorem 3 is proved.

The second statement of Theorem 3 says that the restrictions of $h$ and $\bar{h}$ to the same leaf of $B_{1}$; i.e., the metrics $h_{1}(x)=g_{1} \chi_{2}\left(L_{1}\right)^{-1}$ and $\bar{h}_{1}(x)=$ $\frac{1}{\chi_{2}(0)} g_{1} \chi_{2}\left(L_{1}\right)^{-1}$, are geodesically equivalent. Equivalently, we can reformulate this by saying that $h_{1}$ and $L_{1}$ are compatible on each leaf of the foliation $F_{1}$. To prove 
this fact we only need to notice that on each fixed leaf, $\chi_{2}\left(L_{1}\right)$ is a polynomial (with constant coefficients) in $L_{1}$ and, therefore, $\chi_{2}\left(L_{1}\right)^{-1}$ is a "good" function of $L_{1}$ so that the compatibility of $h_{1}=g_{1} \chi_{2}\left(L_{1}\right)^{-1}$ and $L_{1}$ follows from Theorem 5 and Condition 1 of Proposition 4 .

Proof of Theorem 4. Now we are going to show that the compatibility of the pairs $h_{1}(x), L_{1}(x)$ and $h_{2}(y), L_{2}(y)$ implies the compatibility of

$$
g=\left(\begin{array}{cc}
g_{1}(x, y) & 0 \\
0 & g_{2}(x, y)
\end{array}\right)=\left(\begin{array}{cc}
h_{1} \chi_{2}\left(L_{1}\right) & 0 \\
0 & h_{2} \chi_{1}\left(L_{2}\right)
\end{array}\right) \quad \text { and } \quad L=\left(\begin{array}{cc}
L_{1} & 0 \\
0 & L_{2}
\end{array}\right)
$$

which is equivalent to the statement of Theorem 4

We shall verify the compatibility condition (21) for $u \in D_{1}$ only (the proof for $u \in D_{2}$ is absolutely similar). Since $g$ and $L$ are of block-diagonal form (27), we may use Proposition 4 and replace (21) by Conditions 1-3.

Condition 1 (i.e., compatibility of $g_{1}=h_{1} \chi_{2}\left(L_{1}\right)$ and $L_{1}$ on leaves of $\left.B_{1}\right)$ immediately follows from the Topalov-Sinjukov Theorem and the compatibility of $h_{1}$ and $L_{1}$.

To verify Condition 2, we notice that Lemma 9 and the condition $\frac{\partial}{\partial y^{\alpha}} h_{1}(x)=0$ imply the relation:

$$
g_{1}^{-1} d_{y} g_{1}-\chi_{2}\left(L_{1}\right)^{-1} d_{y} \chi_{2}\left(L_{1}\right)=0 .
$$

By Lemma 11] $\chi_{2}\left(L_{1}\right)^{-1} d_{y} \chi_{2}\left(L_{1}\right)$ satisfies (39); therefore so does $g_{1}^{-1} d_{y} g_{1}$, which exactly gives Condition 2 .

The last relation (34) (Condition 3) is the matrix equation

$$
\left[L_{2}, g_{2}^{-1} \frac{\partial g_{2}}{\partial x^{k}}\right]=0, \quad \text { for any } k=1, \ldots, r .
$$

By our construction, $g_{2}=h_{2} \chi_{1}\left(L_{2}\right)$, where $\chi_{1}\left(L_{2}\right)$ is a polynomial of the form $\chi_{1}\left(L_{2}\right)=a_{0}(x)+a_{1}(x) L_{2}+a_{2}(x) L_{2}^{2}+a_{3}(x) L_{2}^{3}+\ldots$ and neither $h_{2}$ nor $L_{2}$ depend on $x^{k}$. Hence

$$
\begin{aligned}
g_{2}^{-1} \frac{\partial g_{2}}{\partial x^{k}} & =\chi_{1}\left(L_{2}\right)^{-1} h_{2}^{-1} h_{2}\left(\frac{\partial a_{0}}{\partial x^{k}}+\frac{\partial a_{1}}{\partial x^{k}} L_{2}+\frac{\partial a_{2}}{\partial x^{k}} L_{2}^{2}+\frac{\partial a_{3}}{\partial x^{k}} L_{2}^{3}+\ldots\right) \\
& =\chi_{1}\left(L_{2}\right)^{-1}\left(\frac{\partial a_{0}}{\partial x^{k}}+\frac{\partial a_{1}}{\partial x^{k}} L_{2}+\frac{\partial a_{2}}{\partial x^{k}} L_{2}^{2}+\frac{\partial a_{3}}{\partial x^{k}} L_{2}^{3}+\ldots\right)=f\left(L_{2}\right)
\end{aligned}
$$

where $f\left(L_{2}\right)$ is a function of $L_{2}$ (depending on $x$ as a parameter). Thus, (34) holds since $\left[L_{2}, f\left(L_{2}\right)\right]=0$ for every function $f$ (satisfying assumptions (ii)-(V) of Section 1.3). This completes the proof of Theorem 4 .

\section{ACKNOWLEDGEMENT}

The first author thanks I. Zakharevich for useful discussions. The second author thanks Deutsche Forschungsgemeinschaft (Priority Program 1154 - Global Differential Geometry and Research Training Group 1523 - Quantum and Gravitational Fields) for partial financial support, and V. Shevchishin and N. Higham for useful discussions. 
Note added in proof. Two-dimensional version of the projective Lichnerowicz conjecture for closed manifolds was recently proved in [Ma8].

\section{REFERENCES}

[Am1] A. V. Aminova, Pseudo-Riemannian manifolds with general geodesics, Russian Math. Surveys 48 (1993), no. 2, 105-160. MR 1239862

[Am2] A. V. Aminova, Projective transformations of pseudo-Riemannian manifolds. Geometry, 9. J. Math. Sci. (N. Y.) 113(2003), no. 3, 367-470. MR.1965077(2004a:53089)

[Be] E. Beltrami, Resoluzione del problema: riportari $i$ punti di una superficie sopra un piano in modo che le linee geodetische vengano rappresentante da linee rette, Ann. Mat., 1 (1865), no. 7, 185-204.

[BM] A. V. Bolsinov, V. S. Matveev, Geometrical interpretation of Benenti's systems, J. of Geometry and Physics, 44(2003), 489-506, MR1943174

[BMM] R. L. Bryant, G. Manno, V. S. Matveev, A solution of a problem of Sophus Lie: Normal forms of 2-dim metrics admitting two projective vector fields, Math. Ann. 340(2008), no. 2, 437-463. MR2368987 (2008m:53037)

[Ca] L. Carleson, Mergelyan's theorem on uniform polynomial approximation, Math. Scand. 15(1964), 167-175. MR0198209 (33:6368)

[DR] G. de Rham, Sur la reductibilité d'un espace de Riemann, Comment. Math. Helv. 26(1952), 328-344. MR0052177 (14:584a)

[EM] M. Eastwood, V. S. Matveev: Metric connections in projective differential geometry, Symmetries and Overdetermined Systems of Partial Differential Equations (Minneapolis, MN, 2006), 339-351, IMA Vol. Math. Appl., 144(2007), Springer, New York. MR2384718 (2009a:53018)

[Ei] L. P. Eisenhart, Non-Riemannian Geometry, American Mathematical Society Colloquium Publications VIII(1927). MR1466961 (98j:53001)

[Fu] G. Fubini, Sui gruppi transformazioni geodetiche, Mem. Acc. Torino 53(1903), 261-313.

[GWW] G. W. Gibbons, C. M. Warnick, M. C. Werner, Light-bending in Schwarzschild-deSitter: projective geometry of the optical metric, Class. Quant. Grav. 25(2008), 245009 (8 pages). MR2461162

[Haa] J. Haantjes, On $X_{m}$-forming sets of eigenvectors, Nederl. Akad. Wetensch. Proc. Ser. A. 58(1955) = Indag. Math. 17(1955), 158-162. MR0070232 (16:1151a)

[Hal1] G. S. Hall, Some remarks on symmetries and transformation groups in general relativity, Gen. Relativity Gravitation 30(1998), no. 7, 1099-1110. MR.1632505 (99e:83013)

[Hal2] G. S. Hall, Projective symmetry in FRW spacetimes, Classical Quantum Gravity 17(2000), no. 22, 4637-4644. MR.1797960 (2001i:83020)

[Hal3] G. S. Hall, Symmetries and curvature structure in general relativity, World Scientific Lecture Notes in Physics, 46. World Scientific Publishing Co., Inc., River Edge, NJ, 2004. MR2109072 (2005j:83001)

[HL1] G. S. Hall, D. P. Lonie, Projective collineations in spacetimes, Classical Quantum Gravity 12(1995), no. 4, 1007-1020. MR.1330299 (96b:83017)

[HL2] G. S. Hall, D. P. Lonie, The principle of equivalence and projective structure in spacetimes, Classical Quantum Gravity 24(2007), no. 14, 3617-3636. MR2339411 (2008h:53124)

[HL3] G. S. Hall, D. P. Lonie, The principle of equivalence and cosmological metrics, J. Math. Phys. 49(2008), no. 2., 022502 (13 pages). MR.2392851(2009b:53136)

[Hi] N. J. Higham, Functions of matrices. Theory and computation, Society for Industrial and Applied Mathematics (SIAM), Philadelphia, PA, 2008. MR2396439 (2009b:15001)

[KM1] V. Kiosak, V. S. Matveev, Complete Einstein metrics are geodesically rigid, Comm. Math. Phys. 289(1), 383-400, 2009. MR2504854

[KM2] V. Kiosak, V. S. Matveev, Proof of the projective Lichnerowicz conjecture for pseudoRiemannian metrics with degree of mobility greater than two, Comm. Math. Phys. 297 (2010), 401-426. MR2651904

[La] J.-L. Lagrange, Sur la construction des cartes géographiques, Nouveaux Mémoires de l'Académie des Sciences et Bell-Lettres de Berlin, 1779.

[LC] T. Levi-Civita, Sulle transformazioni delle equazioni dinamiche, Ann. di Mat., serie $2^{a}$, 24(1896), 255-300. 
[Lie] S. Lie, Untersuchungen über geodätische Kurven, Math. Ann. 20(1882); Sophus Lie Gesammelte Abhandlungen, Band 2, erster Teil, 267-374. Teubner, Leipzig, 1935.

[MM] G. Manno, V. S. Matveev, 2-dim metrics admitting two projective vector fields near the points where the vector fields are linearly dependent, in preparation.

[MT1] V. S. Matveev, P. J. Topalov, Trajectory equivalence and corresponding integrals, Regular and Chaotic Dynamics, 3(1998), no. 2, 30-45. MR.1693470

[MT2] V. S. Matveev, P. J. Topalov, Quantum integrability for the Beltrami-Laplace operator as geodesic equivalence, Math. Z. 238(2001), 833-866. MR1872577(2002k:58068)

[Ma1] V. S. Matveev, Hyperbolic manifolds are geodesically rigid, Invent. Math. 151(2003), 579-609. MR 1961339 (2004f:53044)

[Ma2] V. S. Matveev, Three-dimensional manifolds having metrics with the same geodesics, Topology 42(2003) no. 6, 1371-1395. MR.1981360

[Ma3] V. S. Matveev, Lichnerowicz-Obata conjecture in dimension two, Comm. Math. Helv. 81(2005) no. 3, 541-570. MR2165202 (2006g:53134)

[Ma4] V. S. Matveev, Beltrami problem, Lichnerowicz-Obata conjecture and applications of integrable systems in differential geometry, Tr. Semin. Vektorn. Tenzorn. Anal. 26(2005), $214-238$.

[Ma5] V. S. Matveev, Proof of projective Lichnerowicz-Obata conjecture, J. Diff. Geom. 75(2007), 459-502. MR2301453 (2007m:53030)

[Ma6] V. S. Matveev, On projectively equivalent metrics near points of bifurcation, In "Topological methods in the theory of integrable systems" (Eds.: Bolsinov A.V., Fomenko A.T., Oshemkov A.A.; Cambridge scientific publishers), pp. 213 - 240. MR2454556

[Ma7] V. S. Matveev, Two-dimensional metrics admitting precisely one projective vector field, to appear in Math. Ann., arXiv:math/0802.2344.

[Ma8] V. S. Matveev, Pseudo-Riemannian metrics on closed surfaces whose geodesic flows admit nontrivial integrals quadratic in momenta, and proof of the projective Obata conjecture for two-dimensional pseudo-Riemannian metrics, J. Math. Soc. Jpn., to appear, arXiv:math/1002.3934.

[Mi1] J. Mikes, Global geodesic mappings and their generalizations for compact Riemannian spaces. Differential geometry and its applications. Proceedings of the 5th international conference, Opava, Czechoslovakia, August 24-28, 1992. Silesian Univ. Math. Publ. 1(1993), 143-149. http://www.emis.de/proceedings/5ICDGA/III/mike.ps . MR.1255535 (94m:53059)

[Mi2] J. Mikes, Geodesic mappings of affine-connected and Riemannian spaces. Geometry, 2, J. Math. Sci. 78(1996), no. 3, 311-333. MR1384327 (97b:53043)

[Pa] P. Painlevé, Sur les intégrales quadratiques des équations de la Dynamique, Compt. Rend., 124(1897), 221-224.

[Pe1] A. Z. Petrov, Einstein spaces, Pergamon Press. XIII, 1969. MR0244912 (39:6225)

[Pe2] A. Z. Petrov, New methods in the general theory of relativity. (in Russian) Izdat. "Nauka", Moscow, 1966. MR0207365 (34:7181)

[Sc] J. A. Schouten, Erlanger Programm und Ubertragungslehre. Neue Gesichtspunkte zur Grundlegung der Geometrie, Rendiconti Palermo 50(1926), 142-169.

[Sh] I. G. Shandra, On the geodesic mobility of Riemannian spaces, Math. Notes 68(2000), no. 3-4, 528-532. MR.1823149 (2002b:53058)

[Si1] N. S. Sinjukov, Geodesic mappings of Riemannian spaces, (in Russian) "Nauka", Moscow, 1979. MR0552022

[Si2] N. S. Sinjukov, On the theory of a geodesic mapping of Riemannian spaces, Dokl. Akad. Nauk SSSR 169(1966), 770-772. MR0202088 (34:1962)

[So] A. S. Solodovnikov, Projective transformations of Riemannian spaces, Uspehi Mat. Nauk (N.S.) 11(1956), no. 4(70), 45-116. MR0084826

[To] P. Topalov, Families of metrics geodesically equivalent to the analogs of the Poisson sphere, J. Math. Phys. 41(2000), no. 11, 7510-7520. MR1788587(2002h:37114)

[We1] H. Weyl, Zur Infinitisimalgeometrie: Einordnung der projektiven und der konformen Auflösung, Nachrichten von der K. Gesellschaft der Wissenschaften zu Göttingen, Mathematisch-Physikalische Klasse, 1921; "Selecta Hermann Weyl", Birkhäuser Verlag, Basel und Stuttgart, 1956.

[We2] H. Weyl, Geometrie und Physik, Die Naturwissenschafter 19(1931), 49-58; "Hermann Weyl Gesammelte Abhandlungen", Band 3, Springer-Verlag, 1968. 
[Wu] H. Wu, On the de Rham decomposition theorem, Illinois J. Math. 8(1964), 291-311. $\operatorname{MR} 0161280(28: 4488)$

School of Mathematics, Loughborough University, Loughborough, LE11 3TU, United KINGDOM

E-mail address: A.Bolsinov@lboro.ac.uk

Institute of Mathematics, Friedrich-Schiller University Jena, 07737 Jena, Germany

E-mail address: vladimir.matveev@uni-jena.de 